TRANSACTIONS OF THE

AMERICAN MATHEMATICAL SOCIETY

Volume 365, Number 7, July 2013, Pages 3643-3673

S 0002-9947(2012)05739-4

Article electronically published on December 27, 2012

\title{
CLOSED ORBITS AND UNIFORM S-INSTABILITY IN GEOMETRIC INVARIANT THEORY
}

\author{
MICHAEL BATE, BENJAMIN MARTIN, GERHARD RÖHRLE, AND RUDOLF TANGE
}

\begin{abstract}
In this paper we consider various problems involving the action of a reductive group $G$ on an affine variety $V$. We prove some general rationality results about the $G$-orbits in $V$. In addition, we extend fundamental results of Kempf and Hesselink regarding optimal destabilizing parabolic subgroups of $G$ for such general $G$-actions.

We apply our general rationality results to answer a question of Serre concerning the behaviour of his notion of $G$-complete reducibility under separable field extensions. Applications of our new optimality results also include a construction which allows us to associate an optimal destabilizing parabolic subgroup of $G$ to any subgroup of $G$. Finally, we use these new optimality techniques to provide an answer to Tits' Centre Conjecture in a special case.
\end{abstract}

\section{Contents}

1. Introduction

2. Notation and preliminaries

2.1. Basic notation

2.2. Non-connected reductive groups

2.3. $G$-varieties

3. Orbits and rationality

4. Uniform $S$-instability

5. Applications to $G$-complete reducibility

5.1. Geometric criteria for $G$-complete reducibility

5.2. Optimal destabilizing parabolic subgroups for subgroups of $G$

5.3. Counterparts for Lie subalgebras

5.4. A special case of the Centre Conjecture

\section{INTRODUCTION}

Let $G$ be a reductive linear algebraic group over an algebraically closed field $k$ and suppose $G$ acts on an affine variety $V$ over $k$. A fundamental problem in geometric invariant theory is to understand the structure of the $G$-orbits $G \cdot v$ and their closures $\overline{G \cdot v}$ for $v \in V$. It is well known that $\overline{G \cdot v}$ is a union of $G$ orbits, exactly one of which is closed. Moreover, the Hilbert-Mumford Theorem

Received by the editors July 1, 2011 and, in revised form, October 28, 2011.

2010 Mathematics Subject Classification. Primary 20G15, 14L24, $20 \mathrm{E} 42$.

Key words and phrases. Reductive group, $G$-variety, closed orbit, uniform instability, optimal cocharacter, Centre Conjecture. 
[15. Thm. 1.4] tells us that if $G \cdot v$ is not closed, then there exists a cocharacter $\lambda$ of $G$ such that $\lim _{a \rightarrow 0} \lambda(a) \cdot v$ exists and lies outside $G \cdot v$. One can associate to $\lambda$ a parabolic subgroup $P_{\lambda}$ of $G$; we call $\lambda$ a destabilizing cocharacter for $v$ and we call $P_{\lambda}$ a destabilizing parabolic subgroup for $v$.

A strengthened version of the Hilbert-Mumford Theorem - due to Kempf [15] and Rousseau [28] - says that there exists a so-called optimal cocharacter $\lambda_{v}$ such that $\lim _{a \rightarrow 0} \lambda_{v}(a) \cdot v$ exists and lies outside $G \cdot v$, and such that $\lambda_{v}$ takes $v$ outside $G \cdot v$ "as fast as possible". This optimality notion has had several applications, including to $G$-complete reducibility [1, 2], 4] and the theory of associated cocharacters for nilpotent elements of Lie $G$ [14, [26]; see [24, p. 64 and App. 2B] for further discussion. Hesselink used optimality to study the nullcone of a rational $G$-module 13.

In this paper we investigate the structure of the orbits when the field $k$ is not algebraically closed. Little seems to be known here. Indeed, one of Kempf's motivations for his optimality construction was to prove a rationality result for destabilizing cocharacters over a perfect field [15, Thm. 4.2]. In Sections 3 and 4 we prove some results in the general setting of geometric invariant theory. In Section [5] we give applications to the theory of $G$-complete reducibility and Tits' Centre Conjecture. Below we describe the contents of the paper in more detail.

It is convenient to extend the concept of orbit closure to the non-algebraically closed case. We say that the $G(k)$-orbit $G(k) \cdot v$ is cocharacter-closed over $k$ if for any $k$-defined cocharacter $\lambda$ of $G$ such that $v^{\prime}:=\lim _{a \rightarrow 0} \lambda(a) \cdot v$ exists, $v^{\prime}$ is $G(k)$-conjugate to $v$ (see Definition [3.8). Clearly, this notion depends only on the $G(k)$-orbit $G(k) \cdot v$ of $v$ and not on $v$ itself. Let $\bar{k}$ denote the algebraic closure of $k$. It follows from the Hilbert-Mumford Theorem that $G \cdot v$ is closed if and only if $G(\bar{k}) \cdot v$ is cocharacter-closed over $\bar{k}$. It is sensible, therefore, to consider the $G(k)$ orbits that are cocharacter-closed over $k$ as a generalization to non-algebraically closed fields $k$ of the closed $G$-orbits. Some of the ideas in Section 3 of this paper were studied by J. Levy in the special case of characteristic 0; cf. [16]. We thank Levy for drawing our attention to [16].

Understanding the structure of the orbits is a delicate problem because the interplay between the $G$-orbits and the $G(k)$-orbits is quite complicated. Let $v \in V(k)$. Suppose first that $G \cdot v$ is not closed and let $S$ be the unique closed $G$-orbit contained in $\overline{G \cdot v}$. It can happen that $G(k) \cdot v$ is cocharacter-closed over $k$, so there need not exist a $k$-defined cocharacter $\lambda$ such that $\lim _{a \rightarrow 0} \lambda(a) \cdot v$ exists and belongs to $S$; indeed, $S$ need not have any $k$-points at all. Now suppose that $G \cdot v$ is closed. If $\lambda$ is a $k$-defined cocharacter, then it can happen that $\lim _{a \rightarrow 0} \lambda(a) \cdot v$ exists but lies outside $G(k) \cdot v$ : in this case, $G(k) \cdot v$ is not cocharacter-closed over $k$. We give concrete examples of these phenomena in Remark 5.10 (see also Question 3.13 ).

Our work on geometric invariant theory has two main strands. Let $v \in V(k)$ and let $\lambda$ be a $k$-defined cocharacter of $G$ such that $v^{\prime}:=\lim _{a \rightarrow 0} \lambda(a) \cdot v$ exists. First we consider the case when $v^{\prime}$ lies in $G(k) \cdot v$. Our main results here are Theorems 3.3 and 3.10, which show that under some additional hypotheses, $v^{\prime}$ lies in $R_{u}\left(P_{\lambda}\right)(k) \cdot v$. Theorem 3.3 was first proved by H. Kraft and J. Kuttler for $k$ algebraically closed of characteristic zero in case $V=G / H$ is an affine homogeneous space; cf. 29, Prop. 2.1.4] or [11, Prop. 2.1.2].

Second, we consider the case when $v^{\prime}$ lies outside $G \cdot v$. We extend work of Kempf and Hesselink on optimality. In [15, Kempf shows that if $v \in V$ is a point whose 
$G$-orbit $G \cdot v$ is not closed, and $S$ is a $G$-stable closed subvariety of $V$ which meets the closure of $G \cdot v$, then there is an optimal class of cocharacters which move $v$ into $S$ (by taking limits). In a similar vein, in [12, Hesselink develops a notion of uniform instability: here the single point $v \in V$ in Kempf's construction is replaced by a subset $X$ of $V$, but the $G$-stable subvariety $S$ is taken to be a single point of $V$. Moreover, Hesselink's results work for arbitrary non-algebraically closed fields. Our constructions, culminating in Theorem 4.5, combine these two ideas within the single framework of uniform $S$-instability, providing a useful extension of these optimality methods in geometric invariant theory.

There is an important open problem which we do not address. We do not deal with the intermediate case when $v^{\prime}$ lies inside $G \cdot v$ but outside $G(k) \cdot v$ : in particular, our optimality results do not give a true generalization of the Hilbert-MumfordKempf-Rousseau optimality theorem to arbitrary $k$. To do this, one would have to answer the following question. Suppose $v \in V(k)$ and there exists a $k$-defined cocharacter $\lambda$ such that $\lim _{a \rightarrow 0} \lambda(a) \cdot v$ exists and lies outside $G(k) \cdot v$. Does there exist an optimal $k$-defined cocharacter which takes $v$ outside $G(k) \cdot v$ as fast as possible? The cocharacter $\lambda_{v}$ described above will not suffice: for instance, if $G \cdot v$ is closed, then $\lambda_{v}$ is not even defined. We plan to return to this question in future work.

The hypothesis that the point $v \in V$ is a $k$-point turns out to be unnecessarily strong, and we can often get away with a weaker condition on the stabilizer $C_{G}(v)$ (see the beginning of Section 3). This is convenient in applications to $G$-complete reducibility (see Remark 5.7).

As well as being of interest in their own right, our general results on $G$-orbits and rationality have applications to the theory of $G$-complete reducibility, introduced by Serre [32] and developed in [1], [2], 4], [5], [17, [18, [19, 30], 31], 33], 34]. In particular, we are able to use them to answer a question of Serre about how $G$ complete reducibility behaves under extensions of fields (Theorem5.11). Our notion of a cocharacter-closed orbit allows us to give a geometric characterization of $G$ complete reducibility over a field $k$ (Theorem [5.9), thereby extending [1, Cor. 3.7]. We use our optimality results to attach to any subgroup $H$ of $G$ an optimal parabolic subgroup of $G$ containing $H$, which is proper if and only if $H$ is not $G$-completely reducible (see Theorem 5.16 and Definition 5.17). This optimal parabolic subgroup provides a very useful tool in the study of subgroups of reductive groups. As an illustration of its effectiveness, we give short proofs of some existing results, and prove a special case of Tits' Centre Conjecture (Theorem 5.31). An important tool, which we introduce in Definition 5.4, is the notion of a generic tuple of a subgroup $H$ of $G$. Replacing generating tuples with generic tuples allows us to avoid many technical problems that arose in our earlier work (see Remark 5.7).

We also refer the reader to [5], where we discuss further consequences of the results of the present paper.

\section{NotATION AND PRELIMINARIES}

2.1. Basic notation. Let $k$ be a field, let $k_{s}$ denote its separable closure, and let $\bar{k}$ denote its algebraic closure. Note that $k_{s}=\bar{k}$ if $k$ is perfect. We denote the Galois group $\operatorname{Gal}\left(k_{s} / k\right)=\operatorname{Gal}(\bar{k} / k)$ by $\Gamma$. We use the notion of a $k$-scheme from [7, AG.11]: a $k$-scheme is a $\bar{k}$-scheme together with a $k$-structure. So $k$-schemes are assumed to be of finite type and reduced separated $k$-schemes are called $k$-varieties. 
Furthermore, a subscheme of a scheme $V$ over $k$ or over $\bar{k}$ is always a subscheme of $V$ as a scheme over $\bar{k}$ and points of $V$ are always closed points of $V$ as a scheme over $\bar{k}$. By "variety" we mean "variety over $\bar{k}$ ". Non-reduced schemes are only used in Section 4 and there they only play a technical rôle; we always formulate our results for $k$-varieties. If $S$ is a subset of a variety, then $\bar{S}$ denotes the closure of $S$.

Now let $V$ be a $k$-variety. If $k_{1} / k$ is an algebraic extension, then we write $V\left(k_{1}\right)$ for the set of $k_{1}$-points of $V$. By a separable point we mean a $k_{s}$-point. If $W$ is a subvariety of $V$, then we set $W\left(k_{1}\right)=W \cap V\left(k_{1}\right)$. Here we do not assume that $W$ is $k$-defined, so $W\left(k_{1}\right)$ can be empty even when $k_{1}=k_{s}$. The Galois group $\Gamma$ acts on $V$; see, e.g., [35, 11.2]. Recall the Galois criterion for a closed subvariety $W$ of $V$ to be $k$-defined: $W$ is $k$-defined if and only if it contains a $\Gamma$-stable set of separable points of $V$ which is dense in $W$ (see [7. Thm. AG.14.4]).

We denote by $\operatorname{Mat}_{m}$ or $\operatorname{Mat}_{m}(k)$ the algebra of $m \times m$ matrices over $k$. The general linear group $\mathrm{GL}_{m}$ acts on $\mathrm{Mat}_{m}$ by conjugation.

Let $H$ be a $k$-defined linear algebraic group. By a subgroup of $H$ we mean a closed subgroup. We let $Z(H)$ denote the centre of $H$ and $H^{0}$ the connected component of $H$ that contains 1 . Recall that $H$ has a $k$-defined maximal torus 7 , 18.2(i) Thm.]. For $K$ a subgroup of $H$, we denote the centralizer of $K$ in $H$ by $C_{H}(K)$ and the normalizer of $K$ in $H$ by $N_{H}(K)$. We denote the group of algebraic automorphisms of $H$ by Aut $H$.

For the set of cocharacters (one-parameter subgroups) of $H$ we write $Y(H)$; the elements of $Y(H)$ are the homomorphisms from the multiplicative group $\bar{k}^{*}$ to $H$. We denote the set of $k$-defined cocharacters by $Y_{k}(H)$. There is a left action of $H$ on $Y(H)$ given by $(h \cdot \lambda)(a)=h \lambda(a) h^{-1}$ for $\lambda \in Y(H), h \in H$ and $a \in \bar{k}^{*}$. The subset $Y_{k}(H)$ is stabilized by $H(k)$.

The unipotent radical of $H$ is denoted by $R_{u}(H)$; it is the maximal connected normal unipotent subgroup of $H$. The algebraic group $H$ is called reductive if $R_{u}(H)=\{1\}$; note that we do not insist that a reductive group is connected.

Let $A$ be an algebraic group, a Lie algebra or an associative algebra. If $n \in \mathbb{N}$ and $\mathbf{x}=\left(x_{1}, \ldots, x_{n}\right) \in A^{n}$, then we say that $\mathbf{x}$ generates $A$ if the $x_{i}$ generate $A$ as an algebraic group (resp. Lie algebra, resp. associative algebra). By this we mean in the algebraic group case that the algebraic subgroup of $A$ generated by the $x_{i}$ is the whole of $A$, and we say that the algebraic group $A$ is topologically finitely generated.

Throughout the paper, $G$ denotes a $k$-defined reductive algebraic group, possibly disconnected. We say an affine $G$-variety $V$ is $k$-defined if both $V$ and the action of $G$ on $V$ are $k$-defined. By a rational $G$-module, we mean a finite-dimensional vector space over $\bar{k}$ with a linear $G$-action. If both $V$ and the action are $k$-defined, then we say the rational $G$-module is $k$-defined.

Suppose $T$ is a maximal torus of $G$. Let $\Psi=\Psi(G, T)$ be the set of roots of $G$ relative to $T$. Let $\alpha \in \Psi$. Then $U_{\alpha}$ denotes the root subgroup of $G$ associated to $\alpha$.

2.2. Non-connected reductive groups. The crucial idea which allows us to deal with non-connected groups is the introduction of so-called Richardson parabolic subgroups ( $R$-parabolic subgroups) of a reductive group $G$. We briefly recall the main definitions and results; for more details and further results, the reader is referred to [1, Sec. 6]. 
Definition 2.1. For each cocharacter $\lambda \in Y(G)$, let

$$
P_{\lambda}=\left\{g \in G \mid \lim _{a \rightarrow 0} \lambda(a) g \lambda(a)^{-1} \text { exists }\right\}
$$

(see Section 2.3 for the definition of limit). Recall that a subgroup $P$ of $G$ is parabolic if $G / P$ is a complete variety. The subgroup $P_{\lambda}$ is parabolic in this sense, but the converse is not true: e.g., if $G$ is finite, then every subgroup is parabolic, but the only subgroup of $G$ of the form $P_{\lambda}$ is $G$ itself. If we define $L_{\lambda}=\left\{g \in G \mid \lim _{a \rightarrow 0} \lambda(a) g \lambda(a)^{-1}=g\right\}$, then $P_{\lambda}=L_{\lambda} \ltimes R_{u}\left(P_{\lambda}\right)$, and we also have $R_{u}\left(P_{\lambda}\right)=\left\{g \in G \mid \lim _{a \rightarrow 0} \lambda(a) g \lambda(a)^{-1}=1\right\}$. The map $c_{\lambda}: P_{\lambda} \rightarrow L_{\lambda}$ given by $c_{\lambda}(g)=\lim _{a \rightarrow 0} \lambda(a) g \lambda(a)^{-1}$ is a surjective homomorphism of algebraic groups with kernel $R_{u}\left(P_{\lambda}\right)$; it coincides with the usual projection $P_{\lambda} \rightarrow L_{\lambda}$. We abuse notation and denote the corresponding map from $P_{\lambda}^{n}$ to $L_{\lambda}^{n}$ by $c_{\lambda}$ as well, for any $n \in \mathbb{N}$. The subgroups $P_{\lambda}$ for $\lambda \in Y(G)$ are called the Richardson parabolic (or $R$-parabolic) subgroups of $G$. Given an R-parabolic subgroup $P$, a Richardson Levi (or R-Levi) subgroup of $P$ is any subgroup $L_{\lambda}$ such that $\lambda \in Y(G)$ and $P=P_{\lambda}$.

If $G$ is connected, then the R-parabolic subgroups (resp. R-Levi subgroups of Rparabolic subgroups) of $G$ are exactly the parabolic subgroups (resp. Levi subgroups of parabolic subgroups) of $G$; indeed, most of the theory of parabolic subgroups and Levi subgroups of connected reductive groups carries over to R-parabolic and R-Levi subgroups of arbitrary reductive groups. In particular, all R-Levi subgroups of an R-parabolic subgroup $P$ are conjugate under the action of $R_{u}(P)$. If $P, Q$ are R-parabolic subgroups of $G$ and $P^{0}=Q^{0}$, then $R_{u}(P)=R_{u}(Q)$.

Lemma 2.2. Let $P, Q$ be R-parabolic subgroups of $G$ with $P \subseteq Q$ and $P^{0}=Q^{0}$, and let $M$ be an $R$-Levi subgroup of $Q$. Then $P \cap M$ is an $R$-Levi subgroup of $P$.

Proof. Fix a maximal torus $T$ of $G$ such that $T \subseteq M$. Then $T \subseteq P$, since $P^{0}=Q^{0}$. There exists a unique R-Levi subgroup $L$ of $P$ such that $T \subseteq L$, [1, Cor. 6.5]. There exists a unique R-Levi subgroup $M^{\prime}$ of $Q$ such that $L \subseteq M^{\prime}$, [1, Cor. 6.6]. Since $M$ is the unique R-Levi subgroup of $Q$ that contains $T$, [1, Cor. 6.5], we must have $M=M^{\prime}$. Hence $L \subseteq P \cap M$. If this inclusion is proper, then $P \cap M$ meets $R_{u}(P)=R_{u}(Q)$ non-trivially, a contradiction. We deduce that $L=P \cap M$.

We now consider some rationality issues. The proof of the next lemma follows immediately from the definitions of limit and of the actions of $\Gamma$ on $k_{s}$-points and on $k_{s}$-defined morphisms.

Lemma 2.3. Let $\lambda \in Y_{k_{s}}(G)$ and let $\gamma \in \Gamma$. Then $P_{\gamma \cdot \lambda}=\gamma \cdot P_{\lambda}$ and $L_{\gamma \cdot \lambda}=\gamma \cdot L_{\lambda}$.

Remark 2.4. If $G$ is connected, then a parabolic subgroup $P$ of $G$ is $k$-defined if and only if $P=P_{\lambda}$ for some $\lambda \in Y_{k}(G)$, 35. Lem. 15.1.2(ii)]. However, the analogous result for R-parabolic subgroups of a non-connected group $G$ is not true in general. To see this, let $T$ be a non-split one-dimensional torus over $k$ and let $F$ be the group of order 2 acting on $T$ by inversion. Then $T$ is a $k$-defined R-parabolic subgroup of the reductive group $G:=F T$, but $T$ is not of the form $P_{\lambda}$ for any $\lambda$ over $k$, because $Y_{k}(G)=\{0\}$. Our next set of results allows us to deal with this problem.

Lemma 2.5. Let $\lambda \in Y(G)$.

(i) If $P_{\lambda}$ is $k$-defined, then so is $R_{u}\left(P_{\lambda}\right)$. Moreover, if $\lambda$ belongs to $Y_{k}(G)$, then $P_{\lambda}, L_{\lambda}$ and the isomorphism $L_{\lambda} \ltimes R_{u}\left(P_{\lambda}\right) \rightarrow P_{\lambda}$ are $k$-defined. 
(ii) Suppose $P_{\lambda}$ is $k$-defined. Then there exists $\mu \in Y_{k}(G)$ such that $P_{\lambda} \subseteq P_{\mu}$ and $P_{\lambda}^{0}=P_{\mu}^{0}$.

(iii) Let $P$ be a $k$-defined $R$-parabolic subgroup. Then any $k$-defined maximal torus of $P$ is contained in a unique $k$-defined $R$-Levi subgroup of $P$ and any two $k$ defined $R$-Levi subgroups of $P$ are conjugate by a unique element of $R_{u}(P)(k)$.

Proof. (i). We have that $P_{\lambda}^{0}$ is $k$-defined, so $R_{u}\left(P_{\lambda}\right)=R_{u}\left(P_{\lambda}^{0}\right)$ is $k$-defined, by [7, Prop. V.20.5]. Now assume that $\lambda \in Y_{k}(G)$. Then $L_{\lambda}=C_{G}\left(\lambda\left(\bar{k}^{*}\right)\right)$ is defined over $k$, by [7, Cor. III.9.2]. Now the multiplication map $G \times G \rightarrow G$ is $k$-defined, so $P_{\lambda}$ is $k$-defined, thanks to [7, Cor. AG.14.5], and the stated isomorphism is then clearly also $k$-defined.

(ii). After conjugating $\lambda$ by an element of $P_{\lambda}$, we may assume that $\lambda \in Y(T)$ for some $k$-defined maximal torus $T$ of $P_{\lambda}$. Since $T$ splits over a finite Galois extension of $k, \lambda$ has only finitely many $\Gamma$-conjugates. Let $\mu \in Y(T)$ be their sum. Since $P_{\lambda}$ is $k$-defined, we have $P_{\gamma \cdot \lambda}=P_{\lambda}$ for all $\gamma \in \Gamma$. By considering the pairings of $\lambda$ and $\mu$ with the coroots of $G$ relative to $T$, we deduce that $P_{\mu}^{0}=P_{\lambda}^{0}$ (cf. [35, 15.1.2]). Using a $G$-equivariant embedding of $G$ acting on itself by conjugation into a finite-dimensional $G$-module, we deduce that $\lim _{a \rightarrow 0} \mu(a) \cdot g$ exists if $\lim _{a \rightarrow 0}(\gamma \cdot \lambda)(a) \cdot g$ exists for all $\gamma \in \Gamma$. So $P_{\lambda} \subseteq P_{\mu}$.

(iii). Because of [7, Prop. V.20.5] and [1, Cors. 6.5, 6.6, 6.7], it is enough to show that the unique R-Levi subgroup of $P$ containing a given $k$-defined maximal torus of $P$ is $k$-defined. (Here the required uniqueness follows from loc. cit.) Let $T$ be a $k$-defined maximal torus of $P$. By the proof of (ii), there exists $\mu \in Y_{k}(T)$ such that $P \subseteq P_{\mu}$ and $P^{0}=P_{\mu}^{0}$. Clearly, $L_{\mu}$ is the R-Levi subgroup of $P_{\mu}$ containing $T$, and it is $k$-defined by (i). The unique R-Levi subgroup of $P$ containing $T$ is $P \cap L_{\mu}$, by Lemma 2.2. Since $P \cap G\left(k_{s}\right)$ and $L_{\mu} \cap G\left(k_{s}\right)$ are $\Gamma$-stable, the same holds for $P \cap L_{\mu} \cap G\left(k_{s}\right)$. So it suffices to show that this set is dense in $P \cap L_{\mu}$. This follows because the components of $P \cap L_{\mu}$ are components of $L_{\mu}$ and the separable points are dense in each component of $L_{\mu}$.

Corollary 2.6. Let $\lambda \in Y_{k}(G)$ and let $\mu \in Y(G)$ such that $P_{\lambda}=P_{\mu}$ and $L_{\mu}$ is $k$-defined. Then there exists $\nu \in Y_{k}(G)$ such that $P_{\lambda}=P_{\nu}$ and $L_{\mu}=L_{\nu}$.

Proof. By Lemma 2.5(iii), there exists $u \in R_{u}\left(P_{\lambda}\right)(k)$ such that $L_{u \cdot \lambda}=u L_{\lambda} u^{-1}=$ $L_{\mu}$, so we can take $\nu=u \cdot \lambda$.

2.3. $G$-varieties. If $G$ acts on a set $V$, then we denote for a subset $S$ of $V$, the pointwise stabilizer $\{g \in G \mid g \cdot s=s$ for all $s \in S\}$ of $S$ in $G$ by $C_{G}(S)$ and the setwise stabilizer $\{g \in G \mid g \cdot S=S\}$ of $S$ in $V$ by $N_{G}(S)$.

Now suppose $G$ acts on an affine variety $V$ and let $v \in V$. Then for each cocharacter $\lambda \in Y(G)$, we can define a morphism of varieties $\phi_{v, \lambda}: \bar{k}^{*} \rightarrow V$ via the formula $\phi_{v, \lambda}(a)=\lambda(a) \cdot v$. If this morphism extends to a morphism $\widehat{\phi}_{v, \lambda}: \bar{k} \rightarrow V$, then we say that $\lim _{a \rightarrow 0} \lambda(a) \cdot v$ exists, and set this limit equal to $\widehat{\phi}_{v, \lambda}(0)$; note that such an extension, if it exists, is necessarily unique.

Let $\lambda \in Y(G)$. Then the set of $v \in V$ such that $\lim _{a \rightarrow 0} \lambda(a) \cdot v$ exists is $P_{\lambda}$-stable and we have

$$
\lim _{a \rightarrow 0} \lambda(a) \cdot(x \cdot v)=c_{\lambda}(x) \cdot\left(\lim _{a \rightarrow 0} \lambda(a) \cdot v\right),
$$


for all $x \in P_{\lambda}$ and $v \in V$. Suppose that the $G$-variety $V$ is $k$-defined. It is easily shown that if $\phi_{v, \lambda}$ is $k$-defined, then $\widehat{\phi}_{v, \lambda}$ is $k$-defined and $\lim _{a \rightarrow 0} \lambda(a) \cdot v \in V(k)$; in particular, this is the case if $\lambda \in Y_{k}(G)$ and $v \in V(k)$.

Remark 2.8. In many of our proofs, we want to reduce the case of a general $(k$ defined) affine $G$-variety $V$ to the case of a ( $k$-defined) rational $G$-module $V_{0}$. Such a reduction is possible, thanks to [15, Lem. 1.1(a)], for example: given $V$, there is a $k$-defined $G$-equivariant embedding of $V$ inside some $V_{0}$. As this situation arises many times in the sequel, we now set up some standard notation which will be in force throughout the paper.

Let $V$ be a rational $G$-module. Given $\lambda \in Y(G)$ and $n \in \mathbb{Z}$, we define

$$
\begin{aligned}
V_{\lambda, n} & :=\left\{v \in V \mid \lambda(a) \cdot v=a^{n} v \text { for all } a \in \bar{k}^{*}\right\}, \\
V_{\lambda, \geq 0} & :=\sum_{n \geq 0} V_{\lambda, n} \quad \text { and } \quad V_{\lambda,>0}:=\sum_{n>0} V_{\lambda, n} .
\end{aligned}
$$

Then $V_{\lambda, \geq 0}$ consists of the vectors $v \in V$ such that $\lim _{a \rightarrow 0} \lambda(a) \cdot v$ exists, $V_{\lambda,>0}$ is the subset of vectors $v \in V$ such that $\lim _{a \rightarrow 0} \lambda(a) \cdot v=0$, and $V_{\lambda, 0}$ is the subset of vectors $v \in V$ such that $\lim _{a \rightarrow 0} \lambda(a) \cdot v=v$. Furthermore, the limit map $v \mapsto \lim _{a \rightarrow 0} \lambda(a) \cdot v$ is nothing but the projection of $V_{\lambda, \geq 0}$ with kernel $V_{\lambda,>0}$ and image $V_{\lambda, 0}$. Of course, similar remarks apply to $-\lambda, V_{\lambda, \leq 0}:=V_{-\lambda, \geq 0}$, and $V_{\lambda,<0}:=V_{-\lambda,>0}$. If the $G$ module $V$ is defined over $k$, then each $V_{\lambda, n}$ and $V_{\lambda,>0}$, etc., is $k$-defined (cf. [7, II.5.2]).

Now let $T$ be a torus in $G$ with $\lambda \in Y(T)$. For $\chi \in X(T)$, let $V_{\chi}$ denote the corresponding weight space of $T$ in $V$. If $v \in V$, then we denote by $v_{\chi}$ the component of $v$ in the weight space $V_{\chi}$ and we put $\operatorname{supp}_{T}(v)=\left\{\chi \in X(T) \mid v_{\chi} \neq 0\right\}$, called the support of $v$ with respect to $T$. Then $V_{\lambda, 0}, V_{\lambda, \geq 0}$ and $V_{\lambda,>0}$ are the direct sums of the subspaces $V_{\lambda,\langle\lambda, \chi\rangle}$, where $\chi \in X(T)$ is such that $\langle\lambda, \chi\rangle=0, \geq 0$ and $>0$, respectively. Furthermore, $v \in V_{\lambda, \geq 0}$ if and only if $\langle\lambda, \chi\rangle \geq 0$ for all $\chi \in \operatorname{supp}_{T}(v)$.

Finally, we recall a standard result [6, Lem. 5.2]. Suppose $T$ is a maximal torus of $G$ with $\lambda \in Y(T)$. Let $\alpha \in \Psi=\Psi(G, T), v \in V_{\lambda, n}$ and $u \in U_{\alpha}$. Then

$$
u \cdot v-v \in \sum_{m \geq 1} V_{\lambda, n+m\langle\lambda, \alpha\rangle} .
$$

Hence, for any $u \in R_{u}\left(P_{\lambda}\right)$ and any $v \in V_{\lambda, \geq 0}$, we have

$$
u \cdot v-v \in V_{\lambda,>0} \text {. }
$$

We continue with some further preliminary results used in the proofs below.

Lemma 2.12. Suppose $G$ acts on an affine variety $V$. Let $v \in V$, let $\lambda \in Y(G)$ and let $u \in R_{u}\left(P_{\lambda}\right)$. Then $\lim _{a \rightarrow 0} \lambda(a) \cdot v$ exists and equals $u \cdot v$ if and only if $u^{-1} \cdot \lambda$ centralizes $v$.

Proof. If $\lim _{a \rightarrow 0} \lambda(a) \cdot v$ exists and equals $u \cdot v$, then $\lambda$ fixes $u \cdot v$ and therefore $u^{-1} \cdot \lambda$ centralizes $v$. Now assume that the latter is the case. Then $\lim _{a \rightarrow 0} \lambda(a) u^{-1} \lambda(a)^{-1}=1$ and $u^{-1} \lambda(a)^{-1} u$ fixes $v$ for all $a \in \bar{k}^{*}$, so

$$
u \cdot v=\left(\lim _{a \rightarrow 0} \lambda(a) u^{-1} \lambda(a)^{-1}\right) \cdot u \cdot v=\lim _{a \rightarrow 0} \lambda(a) \cdot\left(u^{-1} \lambda(a)^{-1} u\right) \cdot v=\lim _{a \rightarrow 0} \lambda(a) \cdot v .
$$


Lemma 2.13. Suppose $G$ acts on an affine variety $V$. Let $v \in V, \lambda \in Y(G)$, such that $v^{\prime}:=\lim _{a \rightarrow 0} \lambda(a) \cdot v$ exists. Furthermore, let $x \in P_{-\lambda}$ and $u \in R_{u}\left(P_{\lambda}\right)$ be such that $x u \cdot v$ is $\lambda\left(\bar{k}^{*}\right)$-fixed. Then $v^{\prime}=u \cdot v$.

Proof. Without loss, we may assume that $V$ is a rational $G$-module (cf. Remark 2.8). Write $x=y l$, where $y \in R_{u}\left(P_{-\lambda}\right)$ and $l \in L_{\lambda}$. Since $V_{\lambda, \leq 0}$ is $P_{-\lambda}$-stable and $y l u \cdot v \in V_{\lambda, 0}$, we have that $l u \cdot v=y^{-1} y l u \cdot v \in V_{\lambda, \leq 0}$. On the other hand, $l u \cdot v \in V_{\lambda, \geq 0}$, since $v \in V_{\lambda, \geq 0}$ and $V_{\lambda, \geq 0}$ is $P_{\lambda}$-stable. So $l u \cdot v \in V_{\lambda, 0}$. It follows that

$$
l u \cdot v=\lim _{a \rightarrow 0} \lambda(a) \cdot l u \cdot v=\lim _{a \rightarrow 0} \lambda(a) l u \lambda(a)^{-1} \cdot \lim _{a \rightarrow 0} \lambda(a) \cdot v=l \cdot v^{\prime} .
$$

So $v^{\prime}=u \cdot v$.

Remark 2.14. The proof of Lemma 2.13 also works if we replace the assumption that $x u \cdot v$ is $\lambda\left(\bar{k}^{*}\right)$-fixed by the weaker assumption that $\lim _{a \rightarrow 0} \lambda(a)^{-1} \cdot(x u \cdot v)$ exists. If $x u \cdot v$ is $\lambda\left(\bar{k}^{*}\right)$-fixed, then we can draw the additional conclusion that $y l u \cdot v=l u \cdot v$, since $R_{u}\left(P_{-\lambda}\right)$ acts trivially on $V_{\lambda, \leq 0} / V_{\lambda,<0}$, by (2.11).

Lemma 2.15. Let $V$ be a rational $G$-module. Let $\lambda, \mu \in Y(G)$ such that $\lambda\left(\bar{k}^{*}\right)$ and $\mu\left(\bar{k}^{*}\right)$ commute. Then for $t \in \mathbb{N}$ sufficiently large, the following hold:

(i) $V_{t \lambda+\mu, \geq 0} \subseteq V_{\lambda, \geq 0}, V_{t \lambda+\mu,>0} \supseteq V_{\lambda,>0}$ and $V_{t \lambda+\mu, 0}=V_{\lambda, 0} \cap V_{\mu, 0}$;

(ii) $P_{t \lambda+\mu} \subseteq P_{\lambda}$ (hence $R_{u}\left(P_{t \lambda+\mu}\right) \supseteq R_{u}\left(P_{\lambda}\right)$ ) and $L_{t \lambda+\mu}=L_{\lambda} \cap L_{\mu}$.

Furthermore, if $t \in \mathbb{N}$ is such that property (i) holds and $v \in V$ is such that $v^{\prime}:=\lim _{a \rightarrow 0} \lambda(a) \cdot v$ and $v^{\prime \prime}:=\lim _{a \rightarrow 0} \mu(a) \cdot v^{\prime}$ exist, then $\lim _{a \rightarrow 0}(t \lambda+\mu)(a) \cdot v$ exists and equals $v^{\prime \prime}$.

Proof. Choose a maximal torus $T$ of $G$ such that $\lambda, \mu \in Y(T)$. Let $\Phi$ be the set of weights of $T$ on $V$. Choose $t \in \mathbb{N}$ large enough such that for any $\chi \in \Phi$ with $\langle\lambda, \chi\rangle \neq 0$, we have that $\langle t \lambda+\mu, \chi\rangle$ is non-zero and has the same sign as $\langle\lambda, \chi\rangle$. Then (i) follows. Part (ii) follows from the argument of the proof of 20 , Prop. 6.7] (increasing $t$ if necessary). Alternatively, it can be deduced from part (i) by embedding $G$ with the conjugation action $G$-equivariantly in a rational $G$ module $W$ and observing that $P_{\nu}=W_{\nu, \geq 0} \cap G$ and $L_{\nu}=W_{\nu, 0} \cap G$.

Now assume that $t \in \mathbb{N}$ is such that (i) holds and let $v \in V$ be such that the limits $v^{\prime}$ and $v^{\prime \prime}$ above exist. Since (i) holds, we have for all $\chi \in \Phi$ that $\langle t \lambda+\mu, \chi\rangle=0$ if and only if $\langle\lambda, \chi\rangle=0$ and $\langle\mu, \chi\rangle=0$. For $\nu \in Y(T)$, let $\Phi_{\nu, \geq 0}$ and $\Phi_{\nu, 0}$ be the sets of weights $\chi \in \Phi$ such that $\langle\nu, \chi\rangle \geq 0$ and $\langle\nu, \chi\rangle=0$, respectively. Then $\operatorname{supp}_{T}(v) \subseteq \Phi_{\lambda, \geq 0}$ and $\operatorname{supp}_{T}(v) \cap \Phi_{\lambda, 0} \subseteq \Phi_{\mu, \geq 0}$. It follows that $\lim _{a \rightarrow 0}(t \lambda+\mu)(a) \cdot v$ exists and equals $v^{\prime \prime}$.

We finish the section with a result that lets us pass from $k$-points to arbitrary points. Let $V$ be a $k$-defined rational $G$-module and let $k_{1} / k$ be a field extension. Let $v \in V\left(k_{1}\right)$ and let $\lambda \in Y_{k}(G)$. Pick a basis $\left(\alpha_{i}\right)_{i \in I}$ for $k_{1}$ over $k$; then we can write $v=\sum_{i \in J} \alpha_{i} v_{i}$ for some finite subset $J$ of $I$ and certain (unique) $v_{i} \in V(k)$. Clearly, we may assume that $J=\{1, \ldots, n\}$ for some $n \in \mathbb{N}$. Set $\mathbf{v}=\left(v_{1}, \ldots, v_{n}\right) \in$ $V^{n}$ and let $G$ act diagonally on $V^{n}$.

Lemma 2.16. With the notation as above, the following hold:

(i) $\lim _{a \rightarrow 0} \lambda(a) \cdot v$ exists if and only if $\lim _{a \rightarrow 0} \lambda(a) \cdot \mathbf{v}$ exists if and only if $\lim _{a \rightarrow 0} \lambda(a) \cdot v_{i}$ exists for each $i$. 
(ii) Suppose the limits in (i) exist. Then for any $g \in G(k)$, we have $v^{\prime}=g \cdot v$ if and only if $\mathbf{v}^{\prime}=g \cdot \mathbf{v}$ if and only if $v_{i}^{\prime}=g \cdot v_{i}$ for each $i$.

Proof. Part (ii) is obvious. In part (i), it follows easily from the definitions of limit and direct product that the second limit exists if and only if the third limit exists. Since $\lambda$ is $k$-defined, $V_{\lambda, \geq 0}$ is $k$-defined, so $V_{\lambda, \geq 0}=\bigoplus_{i \in I} \alpha_{i}\left(V_{\lambda, \geq 0} \cap V(k)\right)$. So $v \in V_{\lambda, \geq 0}$ if and only if $v_{i} \in V_{\lambda, \geq 0}$ for all $i \in J$. Hence the first limit exists if and only if the third limit exists. This completes the proof.

\section{ORBits AND RATionality}

In this section we prove some results about $G(k)$-orbits as indicated in the Introduction. We maintain the notation from the previous sections; recall in particular that $\Gamma=\operatorname{Gal}\left(k_{s} / k\right)$.

Suppose $V$ is a $k$-defined affine $G$-variety. Even when one is interested mainly in rationality questions, one must sometimes consider points $v \in V$ that are not $k$-points. For instance, we often want to prove results about a $k$-defined subgroup $H$ of $G$ by choosing a generating tuple $\mathbf{h}=\left(h_{1}, \ldots, h_{n}\right) \in H^{n}$ for some $n \in \mathbb{N}$, but $H$ need not admit such a tuple with the $h_{i}$ all being $k$-points (for example when $k$ is finite and $H$ is infinite). Fortunately, the weaker property that $C_{G\left(k_{s}\right)}(v)$ is $\Gamma$-stable will often suffice (see Theorem 3.1, for example). We do not require $C_{G}(v)$ to be $k$-defined here; note that even when $v$ is a $k$-point, $C_{G}(v)$ is $k$-closed but need not be $k$-defined. Some of our results hold without any rationality assumptions on $v$ at all (see Theorems 3.3 and 3.10).

Theorem 3.1. Suppose $V$ is a $k$-defined affine $G$-variety. Let $v \in V$ and let $\lambda \in Y_{k}(G)$ be such that $v^{\prime}:=\lim _{a \rightarrow 0} \lambda(a) \cdot v$ exists. If $v^{\prime}$ is $R_{u}\left(P_{\lambda}\right)\left(k_{s}\right)$-conjugate to $v$ and $C_{G\left(k_{s}\right)}(v)$ is $\Gamma$-stable, then $v^{\prime}$ is $R_{u}\left(P_{\lambda}\right)(k)$-conjugate to $v$.

Proof. Since $C_{G^{0}\left(k_{s}\right)}(v)=C_{G\left(k_{s}\right)}(v) \cap G^{0}\left(k_{s}\right)$, we may assume that $G$ is connected. Set $P=P_{\lambda}$. By hypothesis, there exists $u \in R_{u}(P)\left(k_{s}\right)$ such that $v^{\prime}=u \cdot v$. By Lemma 2.12, $\mu:=u^{-1} \cdot \lambda \in Y_{k_{s}}(G)$ centralizes $v$, so $\mu\left(\bar{k}^{*}\right) \subseteq C_{P}(v)$, and $\mu\left(k_{s}^{*}\right) \subseteq C_{G\left(k_{s}\right)}(v) \cap P$. Note that since $u \in P$, we have $P_{\mu}=P$. Let $H$ be the subgroup of $G$ generated by the $\Gamma$-conjugates of $\mu\left(\bar{k}^{*}\right)$; then the union of the $\Gamma$ conjugates of $\mu\left(k_{s}^{*}\right)$ is dense in $H$, so $H$ is closed, connected and $k_{s}$-defined, by [7. AG.14.5, I.2.2], and $H \subseteq P$, since $\mu\left(\bar{k}^{*}\right) \subseteq P$ and $P$ is $\Gamma$-stable. Moreover, since $C_{G\left(k_{s}\right)}(v)$ is $\Gamma$-stable, we can conclude that $H \subseteq C_{P}(v)$. Since $H$ has a $\Gamma$ stable dense set of separable points, $H$ is $k$-defined, and hence contains a $k$-defined maximal torus $S$. There exists $h \in H$ such that $\mu^{\prime}:=h \cdot \mu$ belongs to $Y(S)$; note that $\mu^{\prime}$ centralizes $v$, and since $h \in P$, we deduce that $P_{\mu^{\prime}}=P$. In case $k$ is perfect, $C_{P}(v)$ is $k$-defined, since $C_{P}(v)=C_{G}(v) \cap P$ is $\Gamma$-stable. So in this case we could simply have taken $S$ to be a $k$-defined maximal torus of $C_{P}(v)$.

By [7. Cor. III.9.2], $C_{P}(S)$ is $k$-defined, so it has a $k$-defined maximal torus $T$. Note that $S \subseteq T$, since $S$ commutes with $T$ and $T$ is maximal. There exists a unique $k$-defined Levi subgroup $L$ of $P$ containing $T$, by Lemma 2.5)(iii). But $L_{\mu^{\prime}}$ is a Levi subgroup of $P$ containing $T$, so $L_{\mu^{\prime}}=L$. Thus we have two Levi subgroups $L_{\lambda}$ and $L_{\mu^{\prime}}$ of $P$, both $k$-defined. By Lemma 2.5)(iii), there exists a unique $u_{0} \in R_{u}(P)(k)$ such that $L_{\lambda}=u_{0} L_{\mu^{\prime}} u_{0}{ }^{-1}$. We also have $\mu^{\prime}=h u^{-1} \cdot \lambda$, and since $h u^{-1} \in P$, we can write $h u^{-1}=u_{1} l$ with $u_{1} \in R_{u}(P)$ and $l \in L_{\lambda}$. But $L_{\lambda}$ centralizes $\lambda$, so 
$\mu^{\prime}=u_{1} l \cdot \lambda=u_{1} \cdot \lambda$. So $u_{0}{ }^{-1} L_{\lambda} u_{0}=L_{\mu^{\prime}}=L_{u_{1} \cdot \lambda}=u_{1} L_{\lambda} u_{1}^{-1}$. Since $R_{u}(P)$ acts simply transitively on the set of Levi subgroups of $P$, we must have $u_{1}=u_{0}{ }^{-1}$, and hence $\mu^{\prime}=u_{0}{ }^{-1} \cdot \lambda$. Applying Lemma 2.12 again, we see that $v^{\prime}=u_{0} \cdot v$, because $\mu^{\prime}$ centralizes $v$. This proves the theorem.

Example 3.2. The assumption that $C_{G\left(k_{s}\right)}(v)$ is $\Gamma$-stable in Theorem 3.1 is necessary. For instance, let $G=\mathrm{SL}_{2}$ act on $V=G$ by conjugation. Choose $y \in k_{s} \backslash k$ and $x \in k^{*} \backslash\{ \pm 1\}$. Let

$$
v^{\prime}=\left(\begin{array}{ll}
x & 0 \\
0 & x^{-1}
\end{array}\right) \text { and } v=\left(\begin{array}{ll}
1 & y \\
0 & 1
\end{array}\right)\left(\begin{array}{ll}
x & 0 \\
0 & x^{-1}
\end{array}\right)\left(\begin{array}{ll}
1 & -y \\
0 & 1
\end{array}\right)
$$

and define $\lambda \in Y_{k}(G)$ by $\lambda(a)=\left(\begin{array}{ll}a & 0 \\ 0 & a^{-1}\end{array}\right)$. It is easily seen that $v^{\prime}=\lim _{a \rightarrow 0} \lambda(a) \cdot v$ and that $v^{\prime}$ is $R_{u}\left(P_{\lambda}\right)\left(k_{s}\right)$-conjugate to $v$ but not $R_{u}\left(P_{\lambda}\right)(k)$-conjugate to $v$.

We can now state our first main result.

Theorem 3.3. Suppose $k$ is perfect. Suppose $V$ is a $k$-defined affine $G$-variety and let $v \in V$. Let $\lambda \in Y_{k}(G)$ such that $v^{\prime}:=\lim _{a \rightarrow 0} \lambda(a) \cdot v$ exists and is $G(k)$-conjugate to $v$. Then $v^{\prime}$ is $R_{u}\left(P_{\lambda}\right)(k)$-conjugate to $v$.

Proof. Fix a maximal torus $T$ of $P_{\lambda}$ such that $\lambda \in Y(T)$ and a Borel subgroup $B$ of $P_{\lambda}^{0}$ such that $T \subseteq B$. Let $B^{-}$be the Borel subgroup of $G$ opposite to $B$ with respect to $T$; note that $B^{-} \subseteq P_{-\lambda}$.

We begin with the case that $k$ is algebraically closed. We can assume that $v \neq v^{\prime}$. For $a \in k^{*}$, set $v_{a}=\lambda(a) \cdot v$; then $v_{a} \neq v^{\prime}$ for all $a \in k^{*}$. We show that $v \in R_{u}\left(P_{\lambda}\right) P_{-\lambda}^{0} \cdot v^{\prime}$. Let $\varphi: G \rightarrow G \cdot v^{\prime}$ be the orbit map of $v^{\prime}$. Then $\varphi$ is open, by [7, AG Cor. 18.4]. The set $R_{u}\left(P_{\lambda}\right) P_{-\lambda}^{0}$ contains the big cell $B B^{-} \subseteq G^{0}$, which is an open neighbourhood of 1 in $G$, so $\varphi\left(R_{u}\left(P_{\lambda}\right) P_{-\lambda}^{0}\right)$ contains an open neighbourhood of $\varphi(1)=v^{\prime}$ in $G \cdot v^{\prime}$. The image of $k^{*}$ under the limit morphism $\widehat{\phi}_{v, \lambda}: k \rightarrow V$ meets this neighbourhood, so there exists $a \in k^{*}$ such that $v_{a} \in R_{u}\left(P_{\lambda}\right) P_{-\lambda}^{0} \cdot v^{\prime}$. But $R_{u}\left(P_{\lambda}\right)$ is normal in $P_{\lambda}$ and hence $R_{u}\left(P_{\lambda}\right) P_{-\lambda}^{0}$ is stable under left multiplication by elements of $T$, so we in fact have $v \in R_{u}\left(P_{\lambda}\right) P_{-\lambda}^{0} \cdot v^{\prime}$. Lemmas 2.5 and 2.13 now imply that $v^{\prime}=u \cdot v$ for some $u \in R_{u}\left(P_{\lambda}\right)$. This completes the proof when $k$ is algebraically closed.

Now assume $k$ is perfect. First assume $v \in V(k)$. By the algebraically closed case, we know that $v$ and $v^{\prime}$ are $R_{u}\left(P_{\lambda}\right)$-conjugate. Since $C_{G\left(k_{s}\right)}(v)$ is $\Gamma$-stable, we can apply Theorem 3.1 to deduce that $v$ and $v^{\prime}$ are $R_{u}\left(P_{\lambda}\right)(k)$-conjugate. Now let $v$ be arbitrary. There is no loss in assuming that $V$ is a $k$-defined rational $G$-module (cf. Remark 2.8). Let $\mathbf{v}, \mathbf{v}^{\prime} \in V^{n}$ be as in Lemma 2.16. Then $\lim _{a \rightarrow 0} \lambda(a) \cdot \mathbf{v}=\mathbf{v}^{\prime}$ and $\mathbf{v}$ and $\mathbf{v}^{\prime}$ are $G(k)$-conjugate, so they are $R_{u}\left(P_{\lambda}\right)(k)$-conjugate by the argument above. Lemma 2.16)(ii) implies that $v$ and $v^{\prime}$ are $R_{u}\left(P_{\lambda}\right)(k)$-conjugate, as required.

Remark 3.4. Theorem 3.3 was first proved by H. Kraft and J. Kuttler for $k$ algebraically closed of characteristic zero in case $V=G / H$ is an affine homogeneous space (by a method different from ours); cf. [29, Prop. 2.1.4] or [11, Prop. 2.1.2]. We do not know whether this theorem holds for arbitrary $k$.

The following consequence of Theorem 3.3 is used in the proof of [5, Prop. 3.34]. 
Corollary 3.5. Let $G_{1}$ and $G_{2}$ be reductive groups and let $V$ be an affine $\left(G_{1} \times G_{2}\right)$ variety. Let $v \in V$ and $\lambda_{1} \in Y\left(G_{1}\right)$ and assume that $v^{\prime}:=\lim _{a \rightarrow 0} \lambda_{1}(a) \cdot v$ exists. Then the following hold:

(i) If $v^{\prime}$ is $\left(G_{1} \times G_{2}\right)$-conjugate to $v$, then it is $G_{1}$-conjugate to $v$. In particular, $G_{1} \cdot v$ is closed if $\left(G_{1} \times G_{2}\right) \cdot v$ is.

(ii) Let $\pi: V \rightarrow V / / G_{2}$ be the canonical projection and assume that $\pi^{-1}(\pi(v))=$ $G_{2} \cdot v$. If $\pi\left(v^{\prime}\right)$ is $G_{1}$-conjugate to $\pi(v)$, then $v^{\prime}$ is $G_{1}$-conjugate to $v$.

Proof. (i). By Theorem 3.3, there exists $u \in R_{u}\left(P_{\lambda_{1}}\left(G_{1} \times G_{2}\right)\right)$ such that $v^{\prime}=u \cdot v$. But $R_{u}\left(P_{\lambda_{1}}\left(G_{1} \times G_{2}\right)\right)=R_{u}\left(P_{\lambda_{1}}\left(G_{1}\right)\right) \times\{1\}$, so $v^{\prime}$ is $G_{1}$-conjugate to $v$, as required. The second assertion follows immediately from the Hilbert-Mumford Theorem.

(ii). This follows immediately from (i).

The following example shows that the converse of Corollary 3.5 (i) does not hold in general.

Example 3.6. Let $G=G_{1} \times G_{2}$, where $G_{i}=k^{*}$ for $i=1,2$ (here $k$ is assumed to be algebraically closed). Set $V=k^{2}$, and let $G$ act on $V$ as follows:

$$
\left(t_{1}, t_{2}\right) \cdot\left(x_{1}, x_{2}\right):=\left(t_{1}^{2} t_{2}^{-1} x_{1}, t_{2}^{2} t_{1}^{-1} x_{2}\right),
$$

for $t_{i} \in G_{i}$ and $\left(x_{1}, x_{2}\right) \in V$. Consider the point $(1,1) \in V$. Then the $G_{i}$-orbits of $(1,1)$ are clearly closed, but the $G$-orbit of $(1,1)$ is not closed (if $\lambda \in Y(G)$ is given by $\lambda(a)=(a, a)$, then $\left.\lim _{a \rightarrow 0} \lambda(a) \cdot(1,1)=(0,0)\right)$.

Here is a further consequence of Theorem 3.3 it gives a criterion for determining whether an orbit is closed when $k$ is perfect.

Corollary 3.7. Assume $k$ is perfect. Let $V$ be a $k$-defined affine $G$-variety and let $v \in V$ such that $C_{G\left(k_{s}\right)}(v)$ is $\Gamma$-stable. Suppose there exists $\lambda \in Y_{k}(G)$ such that $v^{\prime}:=\lim _{a \rightarrow 0} \lambda(a) \cdot v$ exists and is not $R_{u}\left(P_{\lambda}\right)(k)$-conjugate to $v$. Then $v^{\prime} \notin G \cdot v$. In particular, $G \cdot v$ is not closed.

Proof. Suppose $v^{\prime}$ is $G$-conjugate to $v$. Then $v^{\prime}$ is $R_{u}\left(P_{\lambda}\right)$-conjugate to $v$, by Theorem 3.3. Since $C_{G\left(k_{s}\right)}(v)$ is $\Gamma$-stable and $k$ is perfect, $v^{\prime}$ is $R_{u}\left(P_{\lambda}\right)(k)$-conjugate to $v$ by Theorem 3.1, a contradiction. Hence $v^{\prime} \notin G \cdot v$, and thus this orbit is not closed.

In order to state our next main result, we need an appropriate extension of the concept of orbit closure to the non-algebraically closed case.

Definition 3.8. Let $V$ be a $k$-defined affine $G$-variety. Let $v \in V$. We say that the $G(k)$-orbit $G(k) \cdot v$ is cocharacter-closed over $k$ if for any $\lambda \in Y_{k}(G)$ such that $v^{\prime}:=\lim _{a \rightarrow 0} \lambda(a) \cdot v$ exists, $v^{\prime}$ is $G(k)$-conjugate to $v$. Note that we do not require $v$ to be a $k$-point of $V$.

Remark 3.9. In what follows we give $V(k)$ the topology induced by the Zariski topology of $V$.

(i). Let $v \in V(k)$. If $k$ is infinite, then $G(k)$ is dense in $G$ [7, V.18.3 Cor.], so $G(k) \cdot v$ is dense in the closure of $G \cdot v$. It follows easily that if $G(k) \cdot v$ is closed in $V(k)$, then $G(k) \cdot v$ is cocharacter-closed over $k$. Corollary 4.11 below now implies that if $k$ is infinite and perfect and $G(k) \cdot v$ is closed in $V(k)$, then $G \cdot v$ is closed. On the other hand, if $k$ is finite, then $G(k) \cdot v$ is a finite subset of $V(k)$ and hence is closed in $V(k)$, even though $G(k) \cdot v$ need not be cocharacter-closed over $k$. 
(ii). If $G(k) \cdot v$ is cocharacter-closed over $k$, then $G(k) \cdot v$ need not be closed in $V(k)$, even if $G \cdot v$ is closed. We give two examples. First, let $k$ be a non-perfect field of characteristic $p>0$. Let $G=\bar{k}^{*}$ acting on $V=\bar{k}^{*}$ by $g \cdot v=g^{p} v$. Give $G$ and $V$ the obvious $k$-structures. Then $G(k) \cdot 1=\left(k^{*}\right)^{p}$ is not closed in $V(k)=k^{*}$, but $G \cdot 1=\bar{k}^{*}$ is closed. Moreover, $G(k) \cdot 1$ is cocharacter-closed over $k$, since the limit $\lim _{a \rightarrow 0} \lambda(a) \cdot 1$ does not exist for any non-trivial $\lambda \in Y(G)$.

Second, let $k=\mathbb{R}$ and let $G=\mathrm{SL}_{2}$ acting on $V=G$ by conjugation. Let

$$
v=\left(\begin{array}{ll}
0 & -1 \\
1 & 0
\end{array}\right), w=\left(\begin{array}{ll}
0 & 1 \\
-1 & 0
\end{array}\right), \text { and } g=\left(\begin{array}{ll}
i & 0 \\
0 & -i
\end{array}\right) .
$$

Then $v, w \in V(\mathbb{R})$ and $w=g \cdot v$, so $v$ and $w$ are $G(\mathbb{C})$-conjugate, but it is easily checked that they are not $G(\mathbb{R})$-conjugate. Hence $w$ lies in the closure in $V(\mathbb{R})$ of $G(\mathbb{R}) \cdot v$, which implies that $G(\mathbb{R}) \cdot v$ is not closed in $V(\mathbb{R})$. But $G \cdot v$ is closed since $v$ is semisimple $([7$, III.9.2 Thm.]), so $G(\mathbb{R}) \cdot v$ is cocharacter-closed over $\mathbb{R}$ by Corollary 3.7

(iii). In [16, Levy investigated a notion similar to our concept of cocharacterclosure in case of a rational $G$-module in characteristic 0. In [16, Thms. 2.1 and 3.1], Levy proved special cases of our Theorem 3.3 .

Our next result says that we can remove the hypothesis that $k$ is perfect in Theorem 3.3 if we assume that $G$ is connected and $G(k) \cdot v$ is cocharacter-closed over $k$.

Theorem 3.10. Suppose $V$ is a $k$-defined affine $G$-variety. Assume that $G$ is connected. Let $v \in V$. Then the following are equivalent:

(i) $G(k) \cdot v$ is cocharacter-closed over $k$;

(ii) for all $\lambda \in Y_{k}(G)$, if $v^{\prime}:=\lim _{a \rightarrow 0} \lambda(a) \cdot v$ exists, then $v^{\prime}$ is $R_{u}\left(P_{\lambda}\right)(k)$-conjugate to $v$.

Proof. It is immediate that (ii) implies (i), so we need to prove that (i) implies (ii). Assume $G(k) \cdot v$ is cocharacter-closed over $k$. Without loss of generality we can assume that $V$ is a $k$-defined rational $G$-module (cf. Remark 2.8). We argue by induction on $\operatorname{dim} V_{\lambda, 0}$ for $\lambda \in Y_{k}(G)$. Suppose $\lambda \in Y_{k}(G)$ and let $v \in V$ such that $v^{\prime}:=\lim _{a \rightarrow 0} \lambda(a) \cdot v$ exists. If $\operatorname{dim} V_{\lambda, 0}=0$, then $V_{\lambda, 0}=0$ and so $v^{\prime}=0$, which forces $v=0$ and we are done. Let $S$ be a maximal $k$-split torus of $G$ with $\lambda \in Y_{k}(S)$, let ${ }_{k} \Psi$ be the set of roots of $G$ relative to $S$ and let ${ }_{k} W=N_{G}(S) / C_{G}(S)$ be the Weyl group over $k$. Any $w \in{ }_{k} W$ has a representative in $N_{G}(S)(k)$; see [7, V.21.2]. We have $C_{G}(S) \subseteq P_{\lambda}$. Fix a minimal $k$-defined parabolic subgroup $P$ of $G$ with $C_{G}(S) \subseteq P \subseteq P_{\lambda}$. Using the notation of [7, V.21.11], the choice of $P$ corresponds to a choice of simple roots ${ }_{k} \Delta \subseteq{ }_{k} \Psi$ and then, $P_{\lambda}={ }_{k} P_{J}$ for a unique subset $J$ of ${ }_{k} \Delta$. Define the subset ${ }_{k} W^{J}$ of ${ }_{k} W$ as in [7, V.21.21], and for each $w \in{ }_{k} W^{J}$ define the subgroup $U_{w}^{\prime}$ of $R_{u}\left(P_{\lambda}\right)$, as in [7, V.21.14]. For each $w \in{ }_{k} W^{J}$, let $\dot{w}$ be a representative of $w$ in $N_{G}(S)(k)$. Then, by [7, V.21.16 and V.21.29] or [9, 3.16 proof], we have

$$
G(k)=\bigcup_{w \in \in_{k} W^{J}} U_{w}^{\prime}(k) \dot{w} P_{\lambda}(k)
$$

and

$$
\dot{w}^{-1} U_{w}^{\prime} \dot{w} \subseteq R_{u}\left(P_{-\lambda}\right) \quad \text { for each } w \in{ }_{k} W^{J}
$$


Since $G(k) \cdot v$ is cocharacter-closed over $k$, there exists $g \in G(k)$ such that $v^{\prime}=g \cdot v$. By (3.11) and Lemma 2.5, we have $g=u^{\prime} \dot{w} l u$ for some $w \in{ }_{k} W^{J}$, $u^{\prime} \in U_{w}^{\prime}(k), l \in L_{\lambda}(k)$ and $u \in R_{u}\left(P_{\lambda}\right)(k)$. Now the argument splits into two cases. Put $n=\dot{w}$.

Case 1. $n$ normalizes $V_{\lambda, 0}$. Then $n^{-1} u^{\prime} n l u \cdot v=n^{-1} \cdot v^{\prime} \in V_{\lambda, 0}$. Furthermore, $n^{-1} u^{\prime} n \in R_{u}\left(P_{-\lambda}\right)$ by (3.12), so $n^{-1} u^{\prime} n l \in P_{-\lambda}$. The desired conclusion follows from Lemma 2.13 .

Case 2. $n$ does not normalize $V_{\lambda, 0}$. Let $\Phi$ be the set of weights of $S$ on $V$ and for $\nu \in Y_{k}(S)$ let $\Phi_{\nu, \geq 0}$ be the set of weights $\chi \in \Phi$ such that $\langle\nu, \chi\rangle \geq 0$. We have $v^{\prime}=u^{\prime} n l u \cdot v$ and therefore $n^{-1} u^{\prime-1} \cdot v^{\prime}=l u \cdot v$. Furthermore, $u^{\prime-1} \cdot v^{\prime}-v^{\prime} \in V_{\lambda,>0}$ by (2.11), whence $\operatorname{supp}_{S}\left(v^{\prime}\right) \subseteq \operatorname{supp}_{S}\left(u^{\prime-1} \cdot v^{\prime}\right)$. Now $n^{-1}$ normalizes $S$, so

$$
n^{-1} \cdot \operatorname{supp}_{S}\left(v^{\prime}\right) \subseteq n^{-1} \cdot \operatorname{supp}_{S}\left(u^{\prime-1} \cdot v^{\prime}\right)=\operatorname{supp}_{S}\left(n^{-1} u^{\prime-1} \cdot v^{\prime}\right) \subseteq \Phi_{\lambda, \geq 0},
$$

since $n^{-1} u^{\prime-1} \cdot v^{\prime}=l u \cdot v \in V_{\lambda, \geq 0}$. It follows that $\operatorname{supp}_{S}\left(v^{\prime}\right) \subseteq n \cdot \Phi_{\lambda, \geq 0}=\Phi_{n \cdot \lambda, \geq 0}$. So $v^{\prime \prime}:=\lim _{a \rightarrow 0}(n \cdot \lambda)(a) \cdot v^{\prime}$ exists. We can choose $\gamma \in Y_{k}(G)$ of the form $\gamma=t \lambda+n \cdot \lambda$ for $t \in \mathbb{N}$ sufficiently large such that the following hold:

(1) $V_{\gamma, 0} \subseteq V_{\lambda, 0}$ and $V_{\gamma, 0} \subseteq V_{n \cdot \lambda, 0}$;

(2) $v^{\prime \prime}=\lim _{a \rightarrow 0} \gamma(a) \cdot v$;

(3) $v^{\prime \prime}=\lim _{a \rightarrow 0} \gamma(a) \cdot v^{\prime}$

(4) $P_{\gamma} \subseteq P_{\lambda}$.

Properties (1), (2) and (4) follow immediately from Lemma 2.15, while (3) follows from $(2)$, since $\lim _{a \rightarrow 0}(n \cdot \lambda)(a) \cdot v^{\prime}=v^{\prime \prime}$ and $\lambda\left(k^{*}\right)$ fixes $v^{\prime}$. If $V_{\lambda, 0}=V_{\gamma, 0}$, then $V_{\lambda, 0} \subseteq V_{n \cdot \lambda, 0}=n \cdot V_{\lambda, 0}$, so $V_{\lambda, 0}=n \cdot V_{\lambda, 0}$, contradicting the fact that $n$ does not normalize $V_{\lambda, 0}$. Hence we must have $\operatorname{dim} V_{\gamma, 0}<\operatorname{dim} V_{\lambda, 0}$. Now $G(k) \cdot v^{\prime}=G(k) \cdot v$ is cocharacter-closed over $k$. So, by the induction hypothesis, $v$ and $v^{\prime}$ are both $R_{u}\left(P_{\gamma}\right)(k)$-conjugate - and hence $P_{\gamma}(k)$-conjugate - to $v^{\prime \prime}$, and hence $v$ and $v^{\prime}$ are $P_{\gamma}(k)$-conjugate. By $(4), v$ and $v^{\prime}$ are $P_{\lambda}(k)$-conjugate. But then they are $R_{u}\left(P_{\lambda}\right)(k)$-conjugate, by Lemmas 2.5 and 2.13 .

In view of Theorems 3.13 .3 and 3.10 , it is natural to ask the following rationality question.

Question 3.13. Let $V$ be a $k$-defined affine $G$-variety. Let $v \in V$ such that $C_{G\left(k_{s}\right)}(v)$ is $\Gamma$-stable. Suppose $k_{1} / k$ is an algebraic extension. Is it true that $G\left(k_{1}\right) \cdot v$ is cocharacter-closed over $k_{1}$ if and only if $G(k) \cdot v$ is cocharacter-closed over $k$ ?

Our final result in this section gives an affirmative answer to the forward implication of Question 3.13 in two instances.

Theorem 3.14. Let $k_{1} / k$ be an algebraic extension of fields and let $V$ be a $k$-defined affine $G$-variety. Let $v \in V$ such that $C_{G\left(k_{s}\right)}(v)$ is $\Gamma$-stable. Suppose that (i) $G$ is connected and $k_{1} / k$ is separable, or (ii) $k$ is perfect. If $G\left(k_{1}\right) \cdot v$ is cocharacter-closed over $k_{1}$, then $G(k) \cdot v$ is cocharacter-closed over $k$.

Proof. Suppose $\lambda \in Y_{k}(G)$ such that $v^{\prime}=\lim _{a \rightarrow 0} \lambda(a) \cdot v$ exists. Then $\lambda \in Y_{k_{1}}(G)$. Since $G\left(k_{1}\right) \cdot v$ is cocharacter-closed over $k_{1}, v^{\prime}$ is $R_{u}\left(P_{\lambda}\right)\left(k_{1}\right)$-conjugate to $v$, by Theorem 3.10 in case (i) and Theorem 3.3 in case (ii). Since $k_{1} / k$ is separable, Theorem 3.1 implies that $v^{\prime}$ is $R_{u}\left(P_{\lambda}\right)(k)$-conjugate to $v$. Hence $G(k) \cdot v$ is cocharacter-closed over $k$, as required. 
Remarks 3.15. (i). If $v \in V(k)$, then the reverse direction holds for $k$ perfect in Theorem 3.14 and the answer to Question 3.13 is yes: this follows from Corollary 4.11 below.

(ii). For arbitrary $k$ it can happen that $G(k) \cdot v$ is not cocharacter-closed over $k$ but $G \cdot v$ is closed or vice versa, even when $v \in V(k)$ (see Remark 5.10, cf. also Remark 4.10(ii)).

\section{UNIFORM $S$-INSTABILITY}

In this section we show that the results of Kempf in [15] extend to uniform instability as defined by W. Hesselink in 12. Since this is a straightforward modification of Kempf's arguments, we only indicate the relevant changes. We point out here that the extension to non-connected $G$ is unnecessary for the results in this section, since they follow immediately from the corresponding statements for $G$ connected. We state the results for $G$ non-connected, because this is more convenient for our applications. As our field $k$ is not necessarily algebraically closed, we restrict to $k$-defined cocharacters of $G$ in Kempf's optimization procedure; cf. [12].

Throughout this section, $G$ is a reductive $k$-defined normal subgroup of a $k$ defined linear algebraic group $G^{\prime}$ which acts on an affine $\bar{k}$-variety $V$, and $S$ is a non-empty $G$-stable closed subvariety of $V$.

Definition 4.1. A $G^{\prime}(k)$-invariant norm on $Y_{k}(G)$ is a non-negative real-valued function \|\| on $Y_{k}(G)$ such that

(i) $\|g \cdot \lambda\|=\|\lambda\|$ for any $g \in G^{\prime}(k)$ and any $\lambda \in Y_{k}(G)$,

(ii) for any $k$-split $k$-defined torus $T$ of $G$, there is a positive definite integervalued form $($,$) on Y_{k}(T)$ such that $(\lambda, \lambda)=\|\lambda\|^{2}$ for any $\lambda \in Y_{k}(T)$.

If $k=\bar{k}$, then we speak of a $G^{\prime}$-invariant norm on $Y(G)$, and in this case we say that \|\| on $Y(G)$ is $k$-defined if it is $\Gamma$-invariant (see [15, Sec. 4]). Note that a $G^{\prime}$-invariant norm \|\| on $Y(G)$ determines a $G^{\prime}(k)$-invariant norm on $Y_{k}(G)$. A $k$-defined $G^{\prime}$-invariant norm on $Y(G)$ always exists, by the argument of [12, 1.4].

Definition 4.2. For each non-empty subset $X$ of $V$, define $\Lambda(X)$ as the set of $\lambda \in$ $Y(G)$ such that $\lim _{a \rightarrow 0} \lambda(a) \cdot x$ exists for all $x \in X$, and put $\Lambda(X, k)=\Lambda(X) \cap Y_{k}(G)$. Extending Hesselink [12, we call $X$ uniformly $S$-unstable if there exists $\lambda \in \Lambda(X)$ such that $\lim _{a \rightarrow 0} \lambda(a) \cdot x \in S$ for all $x \in X$, and we say that such a cocharacter destabilizes $X$ into $S$ or is a destabilizing cocharacter for $X$ with respect to $S$. We call $X$ uniformly $S$-unstable over $k$ if there exists such a $\lambda$ in $\Lambda(X, k)$. We say that $x \in V$ is $S$-unstable over $k$ if $\{x\}$ is uniformly $S$-unstable over $k$. Finally, (uniformly $S$-) unstable without specifying a field always means (uniformly $S$-) unstable over $\bar{k}$. By the Hilbert-Mumford Theorem, $x \in V$ is $S$-unstable if and only if $\overline{G \cdot x} \cap S \neq \varnothing$.

Remark 4.3. Following Hesselink [12, (2.1)], we allow the trivial case that $X \subseteq S$. In this case the optimal class of Definition 4.4 below consists just of the trivial cocharacter $\lambda=0$ and the optimal destabilizing parabolic subgroup of Definition 4.6 is the whole of $G$. Kempf [15, Thm. 3.4] only defines the optimal class and optimal destabilizing parabolic subgroup if $X=\{x\}$ and $x \notin S$ and in this case our definitions coincide with his.

Let $x \in V$ and let $\lambda \in \Lambda(x)$. Let $\varphi: \bar{k} \rightarrow V$ be the morphism $\widehat{\phi}_{x, \lambda}$ from Section 2.3. If $x \notin S$, then the scheme-theoretic inverse image $\varphi^{-1}(S)$ is either 
empty or has affine ring $\bar{k}[T] /\left(T^{m}\right)$ for a unique $m \in \mathbb{N}$, and we define $a_{S, x}(\lambda):=m$ (taking $m$ to be 0 if $\varphi^{-1}(S)$ is empty). If $x \in S$, then we define $a_{S, x}(\lambda):=\infty$. For a non-empty subset $X$ of $V$ and $\lambda \in \Lambda(X)$, we define $a_{S, X}(\lambda):=\min _{x \in X} a_{S, x}(\lambda)$. Note that $a_{S, X}(\lambda)>0$ if and only if $\lim _{a \rightarrow 0} \lambda(a) \cdot x \in S$ for all $x \in X, a_{S, X}(\lambda)=0$ if and only if $\lim _{a \rightarrow 0} \lambda(a) \cdot x \notin S$ for some $x \in X$, and $a_{S, X}(\lambda)=\infty$ if and only if $X \subseteq S$.

Now we choose a $G^{\prime}(k)$-invariant norm \|\| on $Y_{k}(G)$.

Definition 4.4. Let $X$ be a non-empty subset of $V$. If $X \subseteq S$, we put $\Omega(X, S, k)=$ $\{0\}$, where 0 denotes the trivial cocharacter of $G$. Now assume $X \nsubseteq S$. If the function $\lambda \mapsto a_{S, X}(\lambda) /\|\lambda\|$ has a finite strictly positive maximum value on $\Lambda(X, k) \backslash$ $\{0\}$, then we define $\Omega(X, S, k)$ as the set of indivisible cocharacters in $\Lambda(X, k) \backslash\{0\}$ on which this function takes its maximum value. Otherwise we define $\Omega(X, S, k)=$ $\varnothing$. Note that $X$ is uniformly $S$-unstable over $k$ (in the sense of Definition 4.2) provided $\Omega(X, S, k) \neq \varnothing$. The set $\Omega(X, S, k)$ is called the optimal class for $X$ with respect to $S$ over $k$.

We are now able to state and prove the analogue of Kempf's instability theorem ([15, Thm. 4.2]) in this setting.

Theorem 4.5. Let $X$ be a non-empty subset of $V$ which is uniformly S-unstable over $k$. Then $\Omega(X, S, k)$ is non-empty and has the following properties:

(i) $\lim _{a \rightarrow 0} \lambda(a) \cdot x \in S$ for all $\lambda \in \Omega(X, S, k)$ and any $x \in X$.

(ii) For all $\lambda, \mu \in \Omega(X, S, k)$, we have $P_{\lambda}=P_{\mu}$. Let $P(X, S, k)$ denote the unique $R$-parabolic subgroup of $G$ so defined. (Note that $P(X, S, k)$ is $k$-defined by Lemma 2.5.)

(iii) If $g \in G^{\prime}(k)$, then $\Omega(g \cdot X, g \cdot S, k)=g \cdot \Omega(X, S, k)$ and $P(g \cdot X, g \cdot S, k)=$ $g P(X, S, k) g^{-1}$.

(iv) $R_{u}(P(X, S, k))(k)$ acts simply transitively on $\Omega(X, S, k)$ : that is, for each $k$ defined $R$-Levi subgroup $L$ of $P(X, S, k)$, there exists one and only one $\lambda \in$ $\Omega(X, S, k)$ with $L=L_{\lambda}$. Moreover, $N_{G(k)}(X) \subseteq P(X, S, k)(k)$.

Proof. If $X \subseteq S$, then $\Omega(X, S, k)=\{0\}$ and $P(X, S, k)=G$, so all the statements are trivial in this case. Hence we may assume that $X \nsubseteq S$. We have that $G^{0}$ is $k$-defined and, clearly, $Y_{k}(G)=Y_{k}\left(G^{0}\right)$ and $R_{u}\left(P_{\lambda}\right)=R_{u}\left(P_{\lambda}\left(G^{0}\right)\right)$. So we may assume that $G$ is connected. We use Kempf's "state formalism", 15, Sec. 2]. Actually we may consider states as only defined on $k$-split subtori of $G$. First we need an analogue over $k$ of [15, Thm. 2.2]. This is completely straightforward: we simply work with $Y_{k}(G)$ instead of $Y(G)$ and use the conjugacy of the maximal $k$-split tori of $G$ under $G(k)$, [7, V.20.9(ii)], as in [12]. We also use the result that two $k$-defined parabolic subgroups of $G$ have a common maximal $k$-split torus 7 , V.20.7 Prop.].

Next we need a way to associate to a non-empty finite subset $X_{0} \neq\{0\}$ of a rational $G$-module $V_{0}$ a bounded admissible state. This is done as in [12, 2.4]. Then [15, Lem. 3.2] holds with $V$ and $v$ replaced by $V_{0}$ and $X_{0}$, respectively.

Finally, we need to construct two bounded admissible states as in [15, Lem. 3.3]. This is done precisely as in the proof of loc. cit. The embedding of $V$ in a rational $G$-module $V_{0}$ (denoted $V$ in loc. cit.) and the morphism $f: V \rightarrow W, W$ a rational $G$-module, with the scheme-theoretic preimage $f^{-1}(0)$ equal to $S$, can be chosen as in [15, Thm. 3.4]. Let $\Xi$ and $\Upsilon$ be the state of $X$ in $V_{0}$ and the state of $f(X)$ in 
$W$, respectively. Then assertions (i), (ii), (iii) and the first assertion of (iv) follow as in 15 .

The final assertion of (iv) is proved as follows. Fix $\lambda \in \Omega(X, S, k)$. Let $g \in$ $N_{G(k)}(X)$. Then $g \cdot \Omega(X, S, k)=\Omega(g \cdot X, g \cdot S, k)=\Omega(X, S, k)$, by (iii) (note that $g \cdot S=S)$. So $g \cdot \lambda \in \Omega(X, S, k)$. By the first assertion of (iv), $g \cdot \lambda=u \cdot \lambda$ for some $u \in R_{u}(P(X, S, k))(k)$. So $u^{-1} g \in C_{G}\left(\lambda\left(\bar{k}^{*}\right)\right)=L_{\lambda} \subseteq P(X, S, k)$ and therefore $g \in P(X, S, k) \cap G(k)=P(X, S, k)(k)$.

Definition 4.6. We call $P(X, S, k)$ from Theorem 4.5 the optimal destabilizing $R$-parabolic subgroup for $X$ with respect to $S$ over $k$. It is clear that if $P(X, S, k)$ is a proper subgroup of $G$, then $X \nsubseteq S$. If $k$ is algebraically closed, then we often suppress the $k$ argument and write simply $\Omega(X, S)$ and $P(X, S)$.

Next we discuss rationality properties of this construction. If $X$ is uniformly $S$ unstable over $k$ and $k_{1} / k$ is a field extension, then $X$ is uniformly $S$-unstable over $k_{1}$. We want to investigate the relationship between $P(X, S, k)$ and $P\left(X, S, k_{1}\right)$. It appears that one can say little in general if $k_{1} / k$ is not separable, so we consider the special case when $k_{1}=k_{s}$. We denote the $k$-closure of $X$ by $X^{k}$; cf. [7, AG.11.3]. We obtain a rationality result as in [12, Thm. 5.5].

We now choose a $G^{\prime}$-invariant norm \|\| on $Y(G)$. Note that this determines a $G\left(k_{1}\right)$-invariant norm on $Y_{k_{1}}(G)$ for any subfield $k_{1}$ of $\bar{k}$.

Theorem 4.7. Assume that $V$ is an affine $k$-variety and that $S$ and the action of $G$ on $V$ are $k$-defined. Let $X$ be a non-empty subset of $V$. Then the following hold:

(i) $X$ is uniformly $S$-unstable over $k$ if and only if $X^{k}$ is uniformly $S$-unstable over $k_{s}$.

(ii) Assume that $X$ is uniformly $S$-unstable over $k$ and that the norm \|\| on $Y(G)$ is $k$-defined. Then $\Omega(X, S, k)$ consists of the $k$-defined cocharacters in $\Omega\left(X^{k}, S, k_{s}\right)$. In particular, the cocharacters in $\Omega(X, S, k)$ are optimal for $X^{k}$ over $k_{s}$.

Proof. The embedding $V \hookrightarrow V_{0}$ and the morphism $f: V \rightarrow W$ of the proof of Theorem 4.5 can chosen to be defined over $k$; see [7, I.1.9] and the proof of [15, Lem. 1.1]. One can then easily check that for $\lambda \in Y_{k}(G)$ and any integer $r$,

$$
\text { the set }\left\{x \in V \mid \lambda \in \Lambda(x), a_{S, x}(\lambda) \geq r\right\} \text { is } k \text {-closed; }
$$

cf. the proof of [12, Thm. 5.5]. It follows that $\Lambda(X, k)=\Lambda\left(X^{k}, k\right)$ and that $a_{S, X}(\lambda)=a_{S, X^{k}}(\lambda)$ for all $\lambda \in \Lambda(X, k)$.

So we may assume that $X$ is $k$-closed. We have to show that if $X$ is uniformly $S$-unstable over $k_{s}$, then $\Omega\left(X, S, k_{s}\right)$ contains a $k$-defined cocharacter. If $Z$ is a $k$-variety (over $\bar{k}$ ), then $\Gamma=\operatorname{Gal}\left(k_{s} / k\right)$ acts on the set $Z$ and the $k$-closed subsets of $Z$ are the $\Gamma$-stable closed subsets of $Z$; see [35, 11.2.8(ii)]. Furthermore, if $Z_{1}$ and $Z_{2}$ are $k$-varieties, then $\Gamma$ acts on the $k_{s}$-defined morphisms from $Z_{1}$ to $Z_{2}$ and such a morphism is $k$-defined if and only if it is fixed by $\Gamma$; see [35, 11.2.9]. So in our case $\Gamma$ acts on the sets $G$ and $V$ and $X$ is $\Gamma$-stable. Now we can finish the proof as in [15, Thm. 4.2] or [12, Thm. 5.5].

Corollary 4.9. Suppose the hypotheses of Theorem 4.7 hold and that \|\| is $k$ defined. Let $k_{1} / k$ be a separable algebraic extension. Then $X$ is uniformly $S$ unstable over $k$ if and only if $X^{k}$ is uniformly $S$-unstable over $k_{1}$, and in this case we have $\Omega(X, S, k)=\Omega\left(X^{k}, S, k_{1}\right) \cap Y_{k}(G)$ and $P(X, S, k)=P\left(X^{k}, S, k_{1}\right)$. 
Remarks 4.10. (i). Hesselink's optimal class consists in general of virtual cocharacters, since, essentially, he requires $a_{S, X}(\lambda)=1$ (he minimizes the norm). We work with Kempf's optimal class which consists of indivisible cocharacters. There is an obvious bijection between the two optimal classes.

(ii). If $k$ is not perfect, then $X$ can be $S$-unstable over $\bar{k}$ but need not be $S$ unstable over $k$ (see Remark 5.10). Even when $X$ is $S$-unstable over both $k$ and $\bar{k}$, our methods do not tell us whether or not $P(X, S, k)=P(X, S)$ when $k$ is not perfect.

(iii). Assume that $k$ is perfect and that $X=\{v\}$ with $v$ a $k$-point of $V$ outside $S$ whose $G$-orbit closure meets $S$. Then Corollary 4.9 gives the existence of a $k$ defined destabilizing cocharacter for $v$ and $S$ which is optimal over $\bar{k}$. This was first proved by Kempf in [15, Thm. 4.2].

Corollary 4.11 below and Corollary 4.9 answer Question 3.13 for perfect $k$.

Corollary 4.11. Suppose that $k$ is perfect. Let $V$ be an affine $G$-variety over $k$. Let $v \in V(k)$. Then $G \cdot v$ is closed if and only if $G(k) \cdot v$ is cocharacter-closed over $k$.

Proof. If $G(k) \cdot v$ is not cocharacter-closed over $k$, then $G \cdot v$ is not closed, by Corollary 3.7. Conversely, suppose $G \cdot v$ is not closed. Let $S$ be the unique closed $G$-orbit in $\overline{G \cdot v}$. Then $\overline{G \cdot v}$ is $k$-defined (see, e.g., [35, 1.9.1]). Let $\gamma \in \Gamma$. Then $\gamma(S)$ is a closed $G$-orbit which is contained in $\overline{G \cdot v}$, so it is equal to $S$. It follows that $S$ is $\Gamma$-stable and therefore $k$-defined, since $k$ is perfect. Now $v$ is $S$-unstable by the Hilbert-Mumford Theorem and therefore $S$-unstable over $k$, by Theorem 4.7(i). Since $S \cap G \cdot v=\varnothing$, it is clear that $G(k) \cdot v$ is not cocharacter-closed over $k$.

\section{Applications to $G$-COMPlete Reducibility}

In this section we discuss some applications of the theory developed in this paper, with particular reference to Serre's concept of $G$-complete reducibility. We briefly recall the definitions here; for more details, see [1], 34].

Definition 5.1. A subgroup $H$ of $G$ is said to be $G$-completely reducible ( $G$-cr) if whenever $H$ is contained in an R-parabolic subgroup $P$ of $G$, there exists an R-Levi subgroup $L$ of $P$ containing $H$. Similarly, a subgroup $H$ of $G$ is said to be $G$-completely reducible over $k$ if whenever $H$ is contained in a $k$-defined R-parabolic subgroup $P$ of $G$, there exists a $k$-defined R-Levi subgroup $L$ of $P$ containing $H$.

We have noted (Remark 2.4) that not every $k$-defined R-parabolic subgroup of $G$ need stem from a cocharacter in $Y_{k}(G)$. However, our next result shows that when considering questions of $G$-complete reducibility over $k$, it suffices just to look at $k$-defined R-parabolic subgroups of $G$ of the form $P_{\lambda}$ with $\lambda \in Y_{k}(G)$.

Lemma 5.2. Let $H$ be a subgroup of $G$. Then $H$ is $G$-completely reducible over $k$ if and only if for every $\lambda \in Y_{k}(G)$ such that $H$ is contained in $P_{\lambda}$, there exists $\mu \in Y_{k}(G)$ such that $P_{\lambda}=P_{\mu}$ and $H \subseteq L_{\mu}$.

Proof. Assume that for every $\lambda \in Y_{k}(G)$ such that $H$ is contained in $P_{\lambda}$, there exists $\mu \in Y_{k}(G)$ such that $P_{\lambda}=P_{\mu}$ and $H \subseteq L_{\mu}$. Let $\sigma \in Y(G)$ such that $P_{\sigma}$ is $k$-defined and $H \subseteq P_{\sigma}$. After conjugating $\sigma$ by an element of $P_{\sigma}$, we may assume that $\sigma \in Y(T)$ for some $k$-defined maximal torus $T$ of $P_{\sigma}$. By Lemma 2.5(ii), there exists $\lambda \in Y_{k}(T)$ such that $P_{\sigma} \subseteq P_{\lambda}$ and $P_{\sigma}^{0}=P_{\lambda}^{0}$. Note that $L_{\sigma}=L_{\lambda} \cap P_{\sigma}$, 
by Lemma 2.2. By assumption, there exists $\mu \in Y_{k}(G)$ such that $P_{\lambda}=P_{\mu}$ and $H \subseteq L_{\mu}$. There exists $u \in R_{u}\left(P_{\lambda}\right)=R_{u}\left(P_{\sigma}\right)$ such that $u L_{\lambda} u^{-1}=L_{\mu}$. But then $L_{u \cdot \sigma}=u L_{\sigma} u^{-1}=u\left(L_{\lambda} \cap P_{\sigma}\right) u^{-1}=L_{\mu} \cap P_{\sigma}$ contains $H$. By Lemma 2.5(iii), $L_{u \cdot \sigma}$ is $k$-defined, since $L_{u \cdot \sigma}^{0}=L_{\mu}^{0}$ is $k$-defined. Hence $H$ is $G$-cr over $k$. The other implication follows from Corollary 2.6.

Remark 5.3. If $k$ is algebraically closed (or even perfect; see [1, Thm. 5.8]) and $H$ is $k$-defined, then $H$ is $G$-cr over $k$ if and only if $H$ is $G$-cr.

5.1. Geometric criteria for $G$-complete reducibility. In [1, we show that $G$ complete reducibility has a geometric interpretation in terms of the action of $G$ on $G^{n}$, the $n$-fold Cartesian product of $G$ with itself, by simultaneous conjugation. Let $\mathbf{h} \in G^{n}$ and let $H$ be the algebraic subgroup of $G$ generated by $\mathbf{h}$. Then $G \cdot \mathbf{h}$ is closed in $G^{n}$ if and only if $H$ is $G$-cr [1, Cor. 3.7]. To generalize this to subgroups that are not topologically finitely generated, we need the following concept.

Definition 5.4. Let $H$ be a subgroup of $G$ and let $G \hookrightarrow \mathrm{GL}_{m}$ be an embedding of algebraic groups. Then $\mathbf{h} \in H^{n}$ is called a generic tuple of $H$ for the embedding $G \hookrightarrow \mathrm{GL}_{m}$ if $\mathbf{h}$ generates the associative subalgebra of Mat ${ }_{m}$ spanned by $H$. We call $\mathbf{h} \in H^{n}$ a generic tuple of $H$ if it is a generic tuple of $H$ for some embedding $G \hookrightarrow \mathrm{GL}_{m}$.

Clearly, generic tuples exist for any embedding $G \hookrightarrow \mathrm{GL}_{m}$ if $n$ is sufficiently large. The next lemma gives the main properties of generic tuples.

Lemma 5.5. Let $H$ be a subgroup of $G$, let $\mathbf{h} \in H^{n}$ be a generic tuple of $H$ for some embedding $G \hookrightarrow \mathrm{GL}_{m}$ and let $H^{\prime}$ be the algebraic subgroup of $G$ generated by

h. Then we have:

(i) $C_{M}(\mathbf{h})=C_{M}\left(H^{\prime}\right)=C_{M}(H)$ for any subgroup $M$ of $G$;

(ii) $H^{\prime}$ is contained in the same $R$-parabolic and the same $R$-Levi subgroups of $G$ as $H$;

(iii) if $H \subseteq P_{\lambda}$ for some $\lambda \in Y(G)$, then $c_{\lambda}(\mathbf{h})$ is a generic tuple of $c_{\lambda}(H)$ for the given embedding $G \hookrightarrow \mathrm{GL}_{m}$.

Proof. By assumption, $\mathbf{h}$ generates the associative subalgebra $A$ of $\mathrm{Mat}_{m}$ spanned by $H$. For $\lambda \in Y\left(\mathrm{GL}_{m}\right)$ let $\mathcal{P}_{\lambda}$ be the subset of elements $x \in \mathrm{Mat}_{m}$ such that $\lim _{a \rightarrow 0} \lambda(a) \cdot x$ exists and let $\mathcal{L}_{\lambda}$ be the centralizer of $\lambda\left(k^{*}\right)$ in Mat $_{m}$. Denote the limit morphism $\mathcal{P}_{\lambda} \rightarrow \mathcal{L}_{\lambda}$ by $c_{\lambda}$. The well-known characterization of $\mathcal{P}_{\lambda}$ and $\mathcal{L}_{\lambda}$ in terms of flags of subspaces shows that they are subalgebras of Mat $_{m}$ and that $c_{\lambda}$ is a homomorphism of algebras. For $\lambda \in Y(G)$ we have $P_{\lambda}(G)=G \cap \mathcal{P}_{\lambda}$ and $L_{\lambda}(G)=G \cap \mathcal{L}_{\lambda}$.

(i). If a subset $S$ of $\mathrm{Mat}_{m}$ generates the associative subalgebra $E$ of $\mathrm{Mat}_{m}$, then $C_{M}(S)=M \cap C_{\mathrm{Mat}_{m}}(E)$. So $C_{M}(H)=C_{M}\left(H^{\prime}\right)=M \cap C_{\mathrm{Mat}_{m}}(A)=C_{M}(\mathbf{h})$.

(ii). If a subset $S$ of $G$ generates the associative subalgebra $E$ of $\mathrm{Mat}_{m}$, then $S \subseteq P_{\lambda}(G)$ if and only if $E \subseteq \mathcal{P}_{\lambda}$, and $S \subseteq L_{\lambda}(G)$ if and only if $E \subseteq \mathcal{L}_{\lambda}$. This implies the assertion.

(iii). Since $c_{\lambda}: \mathcal{P}_{\lambda} \rightarrow \mathcal{L}_{\lambda}$ is a homomorphism of associative algebras, $c_{\lambda}(\mathbf{h})$ generates the associative subalgebra $c_{\lambda}(A)$ and this is also the associative subalgebra of $\mathrm{Mat}_{m}$ generated by $c_{\lambda}(H)$.

Remark 5.6. If $H$ is a subgroup of $G$ which is topologically generated by a tuple $\mathbf{h} \in H^{n}$, then $\mathbf{h}$ is a generic tuple of $H$ in the sense of Definition 5.4. To see this, 
consider an embedding $G \hookrightarrow \mathrm{GL}_{m}$. Since the minimal polynomial of each $h_{i}$ has non-zero constant term, we can express $h_{i}^{-1}$ as a polynomial in $h_{i}$. Hence, if $A$ is the associative subalgebra of Mat $_{m}$ generated by $\mathbf{h}$, then $A$ contains the inverses of each of the components $h_{i}$, so it contains the subgroup of $\mathrm{GL}_{m}$ generated by $\mathbf{h}$. But $A$ is closed, so it contains $H$.

Remark 5.7. Let $H$ be a $k$-defined subgroup of $G$. Even if $H$ is topologically finitely generated, there need not exist a $k$-defined generating tuple. The notion of a generic tuple lets us get around this problem. Note that if $\mathbf{h}$ is a generic tuple of $H$, then $C_{G\left(k_{s}\right)}(\mathbf{h})$ is $\Gamma$-stable by Lemma $5.5(\mathrm{i})$, which is a sufficient condition for many of the results in Section 3 to hold. Another advantage of generic tuples is that one can extend the action of $S_{n}$ on an $n$-tuple by permutation of the components (cf. [1, Thm. 5.8]) to an action of $\mathrm{GL}_{n}(k)$ (cf. [5, Sec. 3.8]).

The connection between $G$-complete reducibility and $G$-orbits of tuples is made transparent by part (iii) of the following theorem which is, essentially, a consequence of Theorem 3.3. It also shows how statements about generic tuples can be translated back into statements about subgroups of $G$. Note that, in view of Remark [5.6, the final statement of Theorem 5.8(iii) recovers [1, Cor. 3.7].

\section{Theorem 5.8.}

(i) Let $n \in \mathbb{N}$, let $\mathbf{h} \in G^{n}$ and let $\lambda \in Y(G)$ such that $\mathbf{m}:=\lim _{a \rightarrow 0} \lambda(a) \cdot \mathbf{h}$ exists. Then the following are equivalent:

(a) $\mathbf{m}$ is $G$-conjugate to $\mathbf{h}$;

(b) $\mathbf{m}$ is $R_{u}\left(P_{\lambda}\right)$-conjugate to $\mathbf{h}$;

(c) $\operatorname{dim} G \cdot \mathbf{m}=\operatorname{dim} G \cdot \mathbf{h}$.

(ii) Let $H$ be a subgroup of $G$ and let $\lambda \in Y(G)$. Suppose $H \subseteq P_{\lambda}$ and set $M=c_{\lambda}(H)$. Then $\operatorname{dim} C_{G}(M) \geq \operatorname{dim} C_{G}(H)$ and the following are equivalent:

(a) $M$ is $G$-conjugate to $H$;

(b) $M$ is $R_{u}\left(P_{\lambda}\right)$-conjugate to $H$;

(c) $H$ is contained in an $R$-Levi subgroup of $P_{\lambda}$;

(d) $\operatorname{dim} C_{G}(M)=\operatorname{dim} C_{G}(H)$.

(iii) Let $H, \lambda$ and $M$ be as in (ii) and let $\mathbf{h} \in H^{n}$ be a generic tuple of $H$. Then the assertions in (i) are equivalent to those in (ii). In particular, $H$ is $G$ completely reducible if and only if $G \cdot \mathbf{h}$ is closed in $G^{n}$.

Proof. (i). It is obvious that (b) implies (a) and (a) implies (c). It follows immediately from Theorem 3.3 and [7, Prop. I.1.8] that (c) implies (b).

(ii) and (iii). Let $\mathbf{h} \in H^{n}$, let $H^{\prime}$ be the algebraic subgroup of $G$ generated by $\mathbf{h}$ and let $\lambda \in Y(G)$. Then $\lim _{a \rightarrow 0} \lambda(a) \cdot \mathbf{h}$ exists if and only if $H^{\prime} \subseteq P_{\lambda}$. Now assume that $\mathbf{m}=\lim _{a \rightarrow 0} \lambda(a) \cdot \mathbf{h}$ exists. Let $u \in R_{u}\left(P_{\lambda}\right)$. Then $\mathbf{h}=u \cdot \mathbf{m}$ if and only if $u \cdot \lambda$ fixes $\mathbf{h}$ (Lemma 2.12) if and only if $H^{\prime} \subseteq L_{u \cdot \lambda}=u L_{\lambda} u^{-1}$. Pick a generic tuple $\mathbf{h} \in H^{n}$ of $H$ for some $n \in \mathbb{N}$. Then $\mathbf{m}=c_{\lambda}(\mathbf{h})$ is a generic tuple of $M$, by Lemma 5.5(iii). Now the first assertion of (ii) follows from the fact that $\operatorname{dim} G \cdot \mathbf{m} \leq \operatorname{dim} G \cdot \mathbf{h}$ (see [7, Prop. I.1.8]), since $\operatorname{dim} G \cdot \mathbf{h}=\operatorname{dim} G-\operatorname{dim} C_{G}(\mathbf{h})$, which equals $\operatorname{dim} G-\operatorname{dim} C_{G}(H)$ (Lemma[5.5(i)), and likewise for $\mathbf{m}$. Now we prove the equivalences. Clearly, (b) implies (a) and (a) implies (d). Furthermore, we have for $u \in R_{u}\left(P_{\lambda}\right)$ that $H \subseteq L_{u \cdot \lambda}$ if and only if $H=c_{u \cdot \lambda}(H)=u M u^{-1}$. So (b) is equivalent to (c). Now assume that (d) holds. Then $\operatorname{dim} G \cdot \mathbf{m}=\operatorname{dim} G \cdot \mathbf{h}$. So $\mathbf{m}$ is $R_{u}\left(P_{\lambda}\right)$-conjugate to $\mathbf{h}$, by (i), whence $H^{\prime}$ is $R_{u}\left(P_{\lambda}\right)$-conjugate to $c_{\lambda}\left(H^{\prime}\right)$. By the equivalence of (b) and (c) (applied to $\left.H^{\prime}\right), H^{\prime}$ is contained in an R-Levi subgroup 
of $P_{\lambda}$. Since $\mathbf{h}$ is a generic tuple of $H$, (c) holds by Lemma 5.5)(ii). Lemma 5.5)(i) implies that (i)(c) and (ii)(d) are equivalent, so the first assertion of (iii) holds. The final assertion of (iii) follows from the first, Lemma 5.5(ii) and the Hilbert-Mumford Theorem.

We now give a geometric characterization of $G$-complete reducibility over an arbitrary field $k$, using Theorem 3.10 . Note that the subgroup $H$ in Theorem 5.9 need not be $k$-defined. In view of Remark 3.9. Theorem 5.9 in the special case $k=\bar{k}$ yields the final assertion of Theorem 5.8 (iii).

Theorem 5.9. Suppose that $G$ is connected. Let $H$ be a subgroup of $G$ and let $\mathbf{h} \in H^{n}$ be a generic tuple of $H$. Then $H$ is $G$-completely reducible over $k$ if and only if $G(k) \cdot \mathbf{h}$ is cocharacter-closed over $k$.

Proof. Suppose that $G(k) \cdot \mathbf{h}$ is cocharacter-closed over $k$. In order to show that $H$ is $G$-cr over $k$, we just need to consider R-parabolic subgroups of $G$ containing $H$ of the form $P_{\lambda}$ with $\lambda \in Y_{k}(G)$, by Lemma 5.2. Let $\lambda \in Y_{k}(G)$ be such that $P_{\lambda}$ contains $H$. Then $\mathbf{h}^{\prime}:=c_{\lambda}(\mathbf{h})$ exists. Since $G(k) \cdot \mathbf{h}$ is cocharacter-closed over $k$, there exists $u \in R_{u}\left(P_{\lambda}\right)(k)$ such that $\mathbf{h}^{\prime}=u \cdot \mathbf{h}$, by Theorem 3.10. By Lemma 2.12 $u^{-1} \cdot \lambda$ centralizes $\mathbf{h}$. Hence $H \subseteq L_{u^{-1} \cdot \lambda}$. Since $L_{u^{-1} \cdot \lambda}$ is $k$-defined, $H$ is $G$-completely reducible over $k$.

Now assume that $H$ is $G$-completely reducible over $k$. Let $\lambda \in Y_{k}(G)$ such that $\mathbf{h}^{\prime}:=c_{\lambda}(\mathbf{h})$ exists. Then $H \subseteq P_{\lambda}$. So, by hypothesis, there exists a $k$-defined RLevi subgroup $L$ of $P_{\lambda}$ with $H \subseteq L$. By Lemma 2.5(iii), there exists $u \in R_{u}\left(P_{\lambda}\right)(k)$ such that $L=u^{-1} L_{\lambda} u=L_{u^{-1} \cdot \lambda}$. Hence $u^{-1} \cdot \lambda$ centralizes $H$ and so $u^{-1} \cdot \lambda$ centralizes $\mathbf{h}$. Thus, by Lemma 2.12 we have $\mathbf{h}^{\prime}=u \cdot \mathbf{h}$. Consequently, $G(k) \cdot \mathbf{h}$ is cocharacter-closed over $k$.

Remark 5.10. We now provide examples for the failure of Question 3.13 in general. In [4, Ex. 7.22], we give an example of a reductive group $G$ and a subgroup $H$, both $k$-defined, such that $H$ is $G$-completely reducible but not $G$-completely reducible over $k$. Let $\mathbf{h} \in H^{n}$ be a generic tuple of $H$. Then, by Theorem [5.9, $G \cdot \mathbf{h}$ is closed in $G^{n}$ but $G(k) \cdot \mathbf{h}$ is not cocharacter-closed over $k$. Conversely, an example due to McNinch, [1, Ex. 5.11], gives a reductive group $G$ and a subgroup $H$, both $k$-defined, such that $H$ is $G$-completely reducible over $k$ but not $G$-completely reducible, and this implies that there exists a generic tuple $\mathbf{h} \in H^{n}$ for some $n \in \mathbb{N}$ such that $G(k) \cdot \mathbf{h}$ is cocharacter-closed over $k$ but $G \cdot \mathbf{h}$ is not closed. Hence $\mathbf{h}$ is uniformly $S$-unstable over $\bar{k}$ but not uniformly $S$-unstable over $k$, where $S$ is the unique closed $G$-orbit contained in $\overline{G \cdot v}$. In fact, we have $S=\{(1, \ldots, 1)\}$ in this example, so $S$ has a $k$-point. Note that $C_{G\left(k_{s}\right)}(\mathbf{h})$ is $\Gamma$-stable in both cases (this follows from Lemma 5.5(i)), so we have counterexamples to Question 3.13. moreover, in both cases the extension $\bar{k} / k$ is not separable.

We even have an example where $v \in V(k), k$ is infinite, $G \cdot v$ is not closed and $G(k) \cdot v$ is a Zariski-closed subset of $V(k)$. Let $k$ be a separably closed non-perfect field of characteristic 2 and let $G=\mathrm{GL}_{2}$ acting on $V=\mathrm{GL}_{2}$ by conjugation. Choose $a \in k^{1 / 2} \backslash k$. Let $v=\left(\begin{array}{ll}0 & 1 \\ a^{2} & 0\end{array}\right)$ and let $v^{\prime}=\left(\begin{array}{ll}a & 0 \\ 0 & a\end{array}\right)$ (cf. [35, 2.4.11]). It is easily checked that the closure of $G \cdot v$ is $G \cdot v \cup\left\{v^{\prime}\right\}$. Moreover, the orbit map $G \rightarrow G \cdot v, g \mapsto g \cdot v$ is separable (cf. [1, Ex. 3.28 and Rem. 3.31]) and hence is surjective on $k$-points [7, AG.13.2 Thm.]. This implies that $G(k) \cdot v$ is closed 
in $V(k)$ (and hence is cocharacter-closed over $k$, by Remark 3.9(ii)). The unique closed $G$-orbit $S$ contained in $\overline{G \cdot v}$ has no $k$-points - in contrast to the previous example - and it follows that $v$ is uniformly $S$-unstable over $\bar{k}$ but not uniformly $S$-unstable over $k$.

The interpretation of $G$-complete reducibility in terms of orbits allows us to provide a partial answer to a question of Serre; for a more general result, see [5. Thm. 4.13]. Let $k_{1} / k$ be a separable algebraic extension of fields. Serre has asked whether it is the case that a $k$-defined subgroup $H$ of $G$ is $G$-completely reducible over $k$ if and only if it is $G$-completely reducible over $k_{1}$. This was proved in [1, Thm. 5.8] for $k$ perfect by passing back and forth between $k$ and $\bar{k}$ and between $k_{1}$ and $\bar{k}$. In general this approach fails because the extension $\bar{k} / k$ need not be separable; we discuss this further in Example 5.21 below. This shows that even if one is interested only in separable field extensions $k_{1} / k$, problems with inseparability can arise.

We can now answer one direction of Serre's question. Theorem 5.11 gives a group-theoretic analogue of Theorem 3.14

Theorem 5.11. Suppose $k_{1} / k$ is a separable extension of fields. Let $H$ be a $k$-defined subgroup of $G$. If $H$ is $G$-completely reducible over $k_{1}$, then $H$ is $G$ completely reducible over $k$.

Proof. Let $\mathbf{h} \in H^{n}$ be a generic tuple of $H$ for some $n$. Suppose $\lambda \in Y_{k}(G)$ is such that $H \subseteq P_{\lambda}$. Then since $H$ is $G$-cr over $k_{1}$, there exists $u_{1} \in R_{u}\left(P_{\lambda}\right)\left(k_{1}\right) \subseteq$ $R_{u}\left(P_{\lambda}\right)\left(k_{s}\right)$ such that $H \subseteq L_{u_{1}-1 \cdot \lambda}$. Thus, $u_{1}{ }^{-1} \cdot \lambda$ centralizes $H$ and so $u_{1}^{-1} \cdot \lambda$ centralizes $\mathbf{h}$. It thus follows from Lemma 2.12 that $\lim _{a \rightarrow 0} \lambda(a) \cdot \mathbf{h}=u_{1} \cdot \mathbf{h}$. Now $C_{G\left(k_{s}\right)}(\mathbf{h})$ is $\Gamma$-stable by Remark [5.7, so we can apply Theorem 3.1] to conclude that there exists $u \in R_{u}\left(P_{\lambda}\right)(k)$ such that $\lim _{a \rightarrow 0} \lambda(a) \cdot \mathbf{h}=u \cdot \mathbf{h}$. Thus $u^{-1} \cdot \lambda \in Y_{k}(G)$ centralizes $\mathbf{h}$ (Lemma 2.12), whence $u^{-1} \cdot \lambda$ centralizes $H$ (Lemma 5.5(ii)). We therefore have $H \subseteq L_{u^{-1} \cdot \lambda}$, a $k$-defined R-Levi subgroup of $P_{\lambda}$, as required.

Example 5.12. We show that the answer to Serre's question is yes when $G=$ $\mathrm{GL}(V)$, where $V=\bar{k}^{n}$ with the standard $k$-structure $k^{n}$ on $V$. This of course determines the usual $k$-structure on GL( $V)$. Let $H$ be a subgroup of $G$ and let $A$ be its enveloping algebra: that is, the $\bar{k}$-span of $H$ in $\operatorname{End}_{\bar{k}}(V)$. Then $A$ is $k$-defined provided $H$ is. To see this, we exhibit a $\Gamma$-stable, dense subset of separable points in $A$ and for this set we simply take the $k_{s}$-span of $H\left(k_{s}\right)$ in $\operatorname{End}_{\bar{k}}(V)$. As a consequence, we obtain the following characterization of $\mathrm{GL}(V)$-complete reducibility over $k$ under the assumption that $H$ is $k$-defined: $H$ is $\operatorname{GL}(V)$-cr over $k$ if and only if $V(k)=k^{n}$ is a semisimple $A(k)$-module (if and only if $A(k)$ is a semisimple algebra). Here $A(k)$ denotes the algebra of $k$-points of $A$ (this is a $k$-structure on $\left.A: A=\bar{k} \otimes_{k} A(k)\right)$.

Finally, if $H$ and $A$ are as above and $k_{1} \subseteq \bar{k}$ is an algebraic extension of $k$, then $A\left(k_{1}\right)=k_{1} \otimes_{k} A(k)$. It follows from [10, Cor. 69.8 and Cor. 69.10] that $A(k)$ is semisimple if and only if $A\left(k_{1}\right)$ is semisimple, provided $k_{1}$ is a separable extension of $k$. By the above this means that $H$ is $\mathrm{GL}(V)$-cr over $k$ if and only if $H$ is $\mathrm{GL}(V)$-cr over $k_{1}$.

5.2. Optimal destabilizing parabolic subgroups for subgroups of $G$. In this section we assume $G$ is a normal $k$-subgroup of a $k$-defined linear algebraic group $G^{\prime}$. If $G^{\prime}$ is not explicitly given, we just take $G^{\prime}$ to be $G$. We fix a $G^{\prime}$-invariant 
norm \| $\|$ on $Y(G)$; see Definition 4.1. Recall our convention that $P_{\lambda}$ is a subgroup of $G$ : so the optimal destabilizing subgroups defined below are parabolic subgroups of $G$, not of $G^{\prime}$.

Let $H$ be a subgroup of $G$ such that $H$ is not $G$-completely reducible. Suppose there exists $\mathbf{h} \in H^{n}$ such that $H$ is generated by $\mathbf{h}$. Then $G \cdot \mathbf{h}$ is not closed in $G^{n}$, and we can construct the optimal destabilizing parabolic subgroup $P_{\mathbf{h}}=P(\mathbf{h}, S)$ of $G$ for $\mathbf{h}$, where $S$ is the unique closed $G$-orbit contained in $\overline{G \cdot \mathbf{h}}$. Several recent results involving $G$-complete reducibility have rested on this construction [21, [1, Sec. 3, Thm. 5.8], 4, Thm. 5.4(a)]. There are some technical problems in applying it. For instance, if $g \in G$ normalizes $H$, then $g$ need not centralize $\mathbf{h}$ (cf. the proof of [4, Prop. 5.7]).

We now show how to associate an optimal destabilizing R-parabolic subgroup $P(H)$ to $H$ using uniform $S$-instability. This avoids the above problems and yields shorter, cleaner proofs, because we need not deal explicitly with a generating tuple for $H$.

Remark 5.13. We can regard the following construction as a generalization of the Borel-Tits construction [8], which associates to a non-trivial unipotent element $u \in G$ a parabolic subgroup $P_{\mathrm{BT}}$ of $G$ such that $u \in R_{u}\left(P_{\mathrm{BT}}\right)$. More generally, the latter construction associates to a non-reductive subgroup $H$ of $G$ a parabolic subgroup $P_{\mathrm{BT}}$ of $G$ such that $R_{u}(H) \subseteq R_{u}\left(P_{\mathrm{BT}}\right)$. Our construction works for any non- $G$-completely reducible $H$, including the case when $H$ is reductive. Note, however, that if $H$ is non-reductive, then $P_{\mathrm{BT}}$ does not necessarily coincide with $P(H)$ from Definition 5.17, [12, Rem. 8.4].

First we need a preliminary result which gives us a closed $G$-stable subvariety $S_{n}(M)$ of $G^{n}$ to work with. The idea is that the $G$-conjugacy class of a generic tuple of the group $M$ from Proposition 5.14 corresponds to the unique closed $G$-orbit in the $G$-orbit closure of a generic tuple of $H$.

Proposition 5.14. Let $H$ be a subgroup of $G$.

(i) There exists $\lambda \in Y(G)$ and a $G$-completely reducible subgroup $M$ of $G$ such that $H \subseteq P_{\lambda}$ and $c_{\lambda}(H)=M$. Moreover, $M$ is unique up to $G$-conjugacy and its $G$-conjugacy class depends only on the $G$-conjugacy class of $H$.

(ii) Any automorphism of the algebraic group $G$ that stabilizes the $G$-conjugacy class of $H$ stabilizes the $G$-conjugacy class of $M$.

(iii) Any $\gamma \in \Gamma$ that stabilizes the $G$-conjugacy class of $H$ stabilizes the $G$-conjugacy class of $M$.

(iv) If $\mu \in Y(G)$ and $H \subseteq P_{\mu}$, then the procedure described in (i) associates the same $G$-conjugacy class of subgroups to $H$ and $c_{\mu}(H)$.

Proof. (i). Let $P_{\lambda}$ be an R-parabolic subgroup of $G$ which is minimal with respect to containing $H$. Since $H \subseteq c_{\lambda}(H) R_{u}\left(P_{\lambda}\right)$ and $R_{u}\left(P_{\lambda}\right) \subseteq R_{u}(Q)$ for every R-parabolic subgroup $Q$ of $G$ with $Q \subseteq P_{\lambda}$, we have that $P_{\lambda}$ is also minimal with respect to containing $M=c_{\lambda}(H)$. So $M$ is $L_{\lambda}$-irreducible and therefore $G$-cr; see [1, Cor. 6.4, Cor. 3.22].

Now suppose $\lambda, \mu \in Y(G)$ such that $H \subseteq P_{\lambda}$ and $H \subseteq P_{\mu}$, and such that $M_{1}=$ $c_{\lambda}(H)$ and $M_{2}=c_{\mu}(H)$ are $G$-cr. Since $P_{\lambda}$ and $P_{\mu}$ have a maximal torus in common (see, e.g., 7, Cor. IV.14.13]), after possibly replacing $M_{1}$ by an $R_{u}\left(P_{\lambda}\right)$-conjugate and $M_{2}$ by an $R_{u}\left(P_{\mu}\right)$-conjugate, we may assume that $\lambda\left(k^{*}\right)$ and $\mu\left(k^{*}\right)$ commute. Clearly, $P_{\lambda} \cap P_{\mu}$ is stable under $c_{\lambda}$ and $c_{\mu}$. It follows from [1, Lem. 6.2(iii)] that, on 
$P_{\lambda} \cap P_{\mu}$, the composition $c_{\lambda} \circ c_{\mu}=c_{\mu} \circ c_{\lambda}$ is the projection $P_{\lambda} \cap P_{\mu} \rightarrow L_{\lambda} \cap L_{\mu}$ with kernel $R_{u}\left(P_{\lambda} \cap P_{\mu}\right)$. So $c_{\lambda}\left(M_{2}\right)=c_{\mu}\left(M_{1}\right)$. Now $M_{1}$ is $G$-cr, so, by Theorem 5.8 (ii), $M_{1}$ is $R_{u}\left(P_{\mu}\right)$-conjugate to $c_{\mu}\left(M_{1}\right)$. Similarly, $M_{2}$ is $R_{u}\left(P_{\lambda}\right)$-conjugate to $c_{\lambda}\left(M_{2}\right)$. So $M_{1}$ and $M_{2}$ are $G$-conjugate. Finally, we observe that if $H \subseteq P_{\lambda}$ and $g \in G$, then $g H g^{-1} \subseteq P_{g \cdot \lambda}$ and $c_{g \cdot \lambda}\left(g H g^{-1}\right)=g c_{\lambda}(H) g^{-1}$, so the $G$-conjugacy class of $M$ only depends on that of $H$.

(ii). Let $\varphi$ be an automorphism of the algebraic group $G$ that stabilizes the $G$ conjugacy class of $H$ and let $\lambda \in Y(G)$ such that $H \subseteq P_{\lambda}$ and $c_{\lambda}(H)$ is $G$-cr. Then $\varphi(H)$ is $G$-conjugate to $H$ and $\varphi(H) \subseteq P_{\varphi \circ \lambda}$. Now $\varphi\left(c_{\lambda}(H)\right)=c_{\varphi \circ \lambda}(\varphi(H))$ is $G$-conjugate to $c_{\lambda}(H)$ by (i), since $c_{\lambda}(H)$ is $G$-cr.

(iii). Let $\gamma \in \Gamma$ such that $\gamma$ stabilizes the $G$-conjugacy class of $H$ and let $\lambda \in$ $Y(G)$ such that $H \subseteq P_{\lambda}$ and $c_{\lambda}(H)$ is $G$-cr. Then $\gamma \cdot H$ is $G$-conjugate to $H$ and $\gamma \cdot H \subseteq P_{\gamma \cdot \lambda}$. Now $\gamma \cdot M$ is $G$-cr by Lemma 2.3, so $\gamma \cdot M=\gamma \cdot\left(c_{\lambda}(H)\right)=c_{\gamma \cdot \lambda}(\gamma \cdot H)$ is $G$-conjugate to $c_{\lambda}(H)$ by (i).

(iv). Assume that $H \subseteq P_{\mu}$ and let $\lambda \in Y(G)$ such that $H \subseteq P_{\lambda}$ and $c_{\lambda}(H)$ is $G$-cr. After replacing $\lambda$ by a $P_{\lambda}$-conjugate and $\mu$ by a $P_{\mu}$-conjugate, we may assume that $\lambda$ and $\mu$ commute. As in (i), $P_{\lambda} \cap P_{\mu}$ is stable under $c_{\lambda}$ and $c_{\mu}$ and $c_{\lambda}\left(c_{\mu}(H)\right)=c_{\mu}\left(c_{\lambda}(H)\right)$ is $R_{u}\left(P_{\mu}\right)$-conjugate to $c_{\lambda}(H)$ and is $G$-cr, since $c_{\lambda}(H)$ is $G$-cr.

Definition 5.15. Let $M$ be a subgroup of $G$. Given $n \in \mathbb{N}$, set $S_{n}(M):=\overline{G \cdot M^{n}}$, a closed $G$-stable subset of $G^{n}$. Note that $S_{n}(M)$ only depends on the $G$-conjugacy class of $M$. Now suppose there exists $\lambda \in Y_{k}(G)$ such that $H \subseteq P_{\lambda}$ and $M=c_{\lambda}(H)$. Note that if $k$ is algebraically closed, then some subgroup of $G$ in the $G$-conjugacy class attached to $H$ of $G$-cr subgroups of $G$, provided by Proposition 5.14, satisfies this hypothesis. Then we have $c_{\lambda}\left(H^{n}\right) \subseteq M^{n} \subseteq S_{n}(M)$, so $H^{n}$ is uniformly $S_{n}(M)$ unstable over $k$ (in the sense of Definition 4.2).

Theorem 5.16. Let $G, G^{\prime}$ and \|\| be as above. Let $H$ be any subgroup of $G$ and let $n \in \mathbb{N}$ such that $H^{n}$ contains a generic tuple of $H$. Let $M$ be a subgroup of $G$ and suppose that $M=c_{\lambda}(H)$ for some $\lambda \in Y_{k}(G)$ with $H \subseteq P_{\lambda}$. Put $\Omega(H, M, k):=$ $\Omega\left(H^{n}, S_{n}(M), k\right)$. Then the following hold:

(i) $P_{\mu}=P_{\nu}$ for all $\mu, \nu \in \Omega(H, M, k)$. Let $P(H, M, k)$ denote the unique $R$ parabolic subgroup of $G$ so defined. Then we have $H \subseteq P(H, M, k)$ and $R_{u}(P(H, M, k))(k)$ acts simply transitively on $\Omega(H, M, k)$.

(ii) For $g \in G^{\prime}(k)$ we have that $\Omega\left(g H g^{-1}, g M g^{-1}, k\right)=g \cdot \Omega(H, M, k)$ and $P\left(g H g^{-1}, g M g^{-1}, k\right)=g P(H, M, k) g^{-1}$. If $g \in G(k)$ normalizes $H$, then $g \in P(H, M, k)$.

(iii) If $\mu \in \Omega(H, M, k)$, then $\operatorname{dim} C_{G}\left(c_{\mu}(H)\right) \geq \operatorname{dim} C_{G}(M)$. If $M$ is $G$-conjugate to $H$, then $\Omega(H, M, k)=\{0\}$ and $P(H, M, k)=G$. If $M$ is not $G$-conjugate to $H$, then $H$ is not contained in any $R$-Levi subgroup of $P(H, M, k)$.

Proof. (i) and (ii). Clearly, $H^{n}$ is uniformly $S_{n}(M)$-unstable over $k$, so $\Omega(H, M, k)$ is well defined. If $\mu \in \Omega(H, M, k)$, then $\lim _{a \rightarrow 0} \mu(a) \cdot \mathbf{h}$ exists for all $\mathbf{h} \in H^{n}$, so $H \subseteq P_{\mu}=P(H, M, k)$. The rest follows immediately from Theorem 4.5.

(iii). We have $\operatorname{dim} C_{G}(\mathbf{m}) \geq \operatorname{dim} C_{G}(M)$ for all $\mathbf{m} \in G \cdot M^{n}$. Since $\mathbf{m} \mapsto$ $\operatorname{dim} C_{G}(\mathbf{m})$ is upper semi-continuous, cf. [25, Lem. 3.7(c)], this inequality holds for all $\mathbf{m} \in S_{n}(M)$. 
Let $\mu \in \Omega(H, M, k)$. Let $\mathbf{h} \in H^{n}$ be a generic tuple of $H$. Then $c_{\mu}(\mathbf{h})$ is a generic tuple of $c_{\mu}(H)$, by Lemma 5.5(iii). So $\operatorname{dim} C_{G}\left(c_{\mu}(H)\right)=\operatorname{dim} C_{G}\left(c_{\mu}(\mathbf{h})\right) \geq$ $\operatorname{dim} C_{G}(M)$, since $c_{\mu}(\mathbf{h}) \in S_{n}(M)$.

It follows easily from the definitions that $P(H, M, k)=G$ if and only if $\Omega(H, M, k)$ $=\{0\}$ if and only if $H^{n} \subseteq S_{n}(M)$. Clearly, the latter is the case if $M$ is $G$ conjugate to $H$. Now assume that $M$ is not $G$-conjugate to $H$ and pick $\mu \in$ $\Omega(H, M, k)$. Then $\operatorname{dim} C_{G}(M)>\operatorname{dim} C_{G}(H)$, by Theorem 5.8(ii) (applied to $\lambda$ ). So $\operatorname{dim} C_{G}\left(c_{\mu}(H)\right)>\operatorname{dim} C_{G}(H)$ by the above, and $H$ is not contained in any R-Levi subgroup of $P(H, M, k)$, by Theorem 5.8(ii) (applied to $\mu$ ).

Definition 5.17. We call $\Omega(H, M, k)$ the optimal class for $H$ with respect to $M$ over $k$ and we call $P(H, M, k)$ the optimal destabilizing $R$-parabolic subgroup for $H$ with respect to $M$ over $k$. Assume the $G$-conjugacy class given by Proposition 5.14 contains a group $M$ of the form $c_{\lambda}(H)$ for some $\lambda \in Y_{k}(G)$. Then we set $\Omega(H, k):=\Omega(H, M, k)$ and $P(H, k):=P(H, M, k)$. Under this assumption we have, by Proposition 5.14 and Theorem 5.16, that $N_{G(k)}(H)$ is contained in $P(H, k)$ and that for $\mu \in \Omega(H, k), c_{\mu}(H)$ is $G$-completely reducible. So, by Theorem 5.8 (ii), if $H$ is not $G$-completely reducible, then it is not contained in any R-Levi subgroup of $P(H, k)$. Note that, trivially, $P(H, k)=G$ if $H$ is $G$-completely reducible. We call $\Omega(H, k)$ the optimal class for $H$ over $k$ and we call $P(H, k)$ the optimal destabilizing R-parabolic subgroup for $H$ over $k$. We suppress the dependence on the choice of $n$ and \|\| in the notation (cf. Remark 5.22).

Note that the assumption of the previous paragraph is satisfied if $k$ is algebraically closed. In that case we usually suppress the $k$ argument and write simply $\Omega(H)$ and $P(H)$ instead; we refer to these as the optimal class for $H$ and the optimal destabilizing $R$-parabolic subgroup for $H$, respectively.

We now suppose that the fixed norm \|\| on $Y(G)$ is $k$-defined; cf. Definition 4.1 . We get the following rationality result.

Theorem 5.18. Let $G, G^{\prime}, H$ and $n$ be as in Theorem 5.16 and assume that $H$ is $k$-closed. Then the following hold:

(i) Suppose that $M$ is a subgroup of $G$ such that $M=c_{\lambda}(H)$ for some $\lambda \in Y_{k_{s}}(G)$ with $H \subseteq P_{\lambda}$ and such that $S_{n}(M)$ is $k$-defined (this is the case in particular if $M$ is $k$-defined $)$. Then $\Omega(H, M, k)$ is well defined and equal to $\Omega\left(H, M, k_{s}\right) \cap$ $Y_{k}(G)$. Moreover, $P(H, M, k)$ is well defined and equal to $P\left(H, M, k_{s}\right)$. In particular, $P\left(H, M, k_{s}\right)$ is $k$-defined.

(ii) If $k$ is perfect, then $\Omega(H, k)$ is well defined and equal to $\Omega(H) \cap Y_{k}(G)$. Moreover, $P(H, k)$ is well defined and equal to $P(H)$. In particular, $P(H)$ is $k$-defined.

Proof. (i). This follows immediately from Theorem 4.7

(ii). Since $k$ is perfect, $k_{s}=\bar{k}$. Let $M$ be as in Proposition 5.14 then $H$ is uniformly $S_{n}(M)$-unstable over $\bar{k}$. Now $S_{n}(M)$ is $\Gamma$-stable by Proposition 5.14(iii) and hence is $k$-defined, since $k$ is perfect. The result now follows from (i).

Remarks 5.19. (i). Let $M$ be as in Theorem 5.16 and let $M_{0}$ be a $G$-cr subgroup from the $G$-conjugacy class associated to $H$ by Proposition 5.14. Then we have $S_{n}\left(M_{0}\right) \subseteq S_{n}(M)$ for any $n$. To prove this we may, by the final assertion in 
Proposition 5.14, assume that $M=H$. Furthermore, we may assume that $M_{0}=$ $c_{\lambda}(H)$ for some $\lambda \in Y(G)$ with $H \subseteq P_{\lambda}$. Since $\lambda(a) \cdot H^{n} \subseteq G \cdot H^{n}$ for all $a \in k^{*}$, we have $M_{0}^{n}=c_{\lambda}\left(H^{n}\right) \subseteq S_{n}(H)$. So $S_{n}\left(M_{0}\right) \subseteq S_{n}(H)$.

(ii). Note that $G \cdot M^{n}$ need not be closed: e.g., take $G$ connected and non-abelian, $n$ to be 1 and $H=M$ to be a maximal torus of $G$.

Example 5.20. We give an example of the usefulness of this construction (cf. 21] and [1, Thm. 3.10]). Let $H$ be a $G$-completely reducible subgroup of $G$ and let $N$ be a normal subgroup of $H$. We prove that $N$ is $G$-completely reducible. Suppose not. Then $H \subseteq N_{G}(N) \subseteq P(N)$. Since $N$ is not contained in an R-Levi subgroup of $P(N)$, neither is $H$. But $H$ is assumed to be $G$-completely reducible, so this is impossible. We deduce that $N$ is $G$-completely reducible after all.

Here is a second example, which illustrates the gap in the theory pointed out in the Introduction.

Example 5.21. Assume $k$ is perfect and $H$ is a $k$-defined subgroup of $G$. Suppose $H$ is not $G$-completely reducible. Then $H$ is not contained in any R-Levi subgroup of the optimal destabilizing R-parabolic subgroup $P(H)$ of $G$. Now $P(H)=P_{\lambda}$ for some $\lambda \in Y_{k}(G)$, by Theorem 5.18 (ii), so $H$ is not $G$-completely reducible over $k$. This proves the forward direction of [1, Thm. 5.8]. The proof of the reverse direction given in loc. cit. is essentially just a special case of the proof of Theorem 3.1.

One deduces from the above as in [1, Thm. 5.8] that if $k_{1} / k$ is a separable algebraic extension of fields and $G$ and $H$ are $k$-defined, then, under the hypothesis that $k$ is perfect, $H$ is $G$-completely reducible over $k_{1}$ if and only if $H$ is $G$-completely reducible over $k$. We answered the forward direction of Serre's question, Theorem 5.11. without the hypothesis that $k$ is perfect. We cannot answer the reverse direction by passing to $\bar{k}$ using the argument in the previous paragraph: for $H$ can be $G$-completely reducible over $k$ (or $k_{1}$ ) and yet not $G$-completely reducible over $\bar{k}$, or vice versa (see Remark 5.10). To give a direct proof that the reverse implication holds, one would like to associate an "optimal destabilizing R-parabolic subgroup" $P$ to $H$ having the property that $P$ is defined over $k_{1}$ and no R-Levi $k_{1}$-subgroup of $P$ contains $H$; optimality should imply that $P$ is $\operatorname{Gal}\left(k_{1} / k\right)$-stable and hence $k$ defined, which would show that $H$ is not $G$-completely reducible over $k$. We cannot take $P$ to be $P\left(H, c_{\mu}(H), k_{1}\right)$ for any $\mu \in Y_{k_{1}}(H)$, because if $H$ is $G$-completely reducible, then $P\left(H, c_{\mu}(H), k_{1}\right)$ is just $G$.

Remark 5.22. The construction of the optimal class of $k$-defined cocharacters and the optimal destabilizing R-parabolic subgroup $P(H)$ from Definition 5.17 depends on the choice of $n$ and the choice of norm \|\| . In view of [12, Sec. 7], it is plausible that this construction is independent of these choices. Since the results we obtain here are sufficient for our applications in the present and subsequent sections, we do not pursue this question here and leave it instead to a future study.

5.3. Counterparts for Lie subalgebras. There are counterparts to our results for Lie subalgebras $\mathfrak{h}$ of the Lie algebra $\mathfrak{g}=\operatorname{Lie} G$ of $G$. All of our results carry over with obvious modifications. For instance, if $\mathfrak{h}$ is not $G$-completely reducible, then there is an optimal destabilizing parabolic subgroup $P$ of $G$ such that $\mathfrak{h} \subseteq \mathfrak{p}$ but $\mathfrak{h} \not \mathfrak{l}$ for any R-Levi subgroup $L$ of $P$; see Theorem 5.27 below. Many of the proofs are actually easier in the Lie algebra case: for example, it often suffices to work in connected $G$. We just state the counterparts of Theorems 5.8 and 5.16 in this Lie algebra setting. We leave the details of the proofs to the reader. 
For a subgroup $H$ of $G$ we denote its Lie algebra Lie $H$ by $\mathfrak{h}$. We start with the analogue of Definition 5.1 in this setting; cf. 222 and also [5, Sec. 3.3].

Definition 5.23. A subalgebra $\mathfrak{h}$ of $\mathfrak{g}$ is $G$-completely reducible if for any $\mathrm{R}$ parabolic subgroup $P$ of $G$ such that $\mathfrak{h} \subseteq \mathfrak{p}$, there is an R-Levi subgroup $L$ of $P$ such that $\mathfrak{h} \subseteq \mathfrak{l}$.

We require some standard facts concerning Lie algebras of R-parabolic and RLevi subgroups of $G$ (cf. [27, Sec. 2.1]).

Lemma 5.24. For $\lambda \in Y(G)$, put $\mathfrak{p}_{\lambda}=\operatorname{Lie}\left(P_{\lambda}\right)$ and $\mathfrak{l}_{\lambda}=\operatorname{Lie}\left(L_{\lambda}\right)$. Let $x \in \mathfrak{g}$. Then

(i) $x \in \mathfrak{p}_{\lambda}$ if and only if $\lim _{a \rightarrow 0} \lambda(a) \cdot x$ exists;

(ii) $x \in \mathfrak{l}_{\lambda}$ if and only if $\lim _{a \rightarrow 0} \lambda(a) \cdot x$ exists and equals $x$ if and only if $\lambda(\bar{k})$ centralizes $x$;

(iii) $x \in \operatorname{Lie}\left(R_{u}\left(P_{\lambda}\right)\right)$ if and only if $\lim _{a \rightarrow 0} \lambda(a) \cdot x$ exists and equals 0 .

The map $c_{\lambda}: \mathfrak{p}_{\lambda} \rightarrow \mathfrak{l}_{\lambda}$ given by $x \mapsto \lim _{a \rightarrow 0} \lambda(a) \cdot x$ coincides with the usual projection of $\mathfrak{p}_{\lambda}$ onto $\mathfrak{l}_{\lambda}$. In analogy with the construction for subgroups of $G$, we consider the action of $G$ on $\mathfrak{g}^{n}$ by simultaneous adjoint action.

Remark 5.25. A statement analogous to Proposition 5.14 holds for Lie algebras: that is, given any Lie subalgebra $\mathfrak{h}$ of $\mathfrak{g}$, we can find a uniquely defined $G$-conjugacy class of subalgebras of $\mathfrak{g}$ which contains $c_{\lambda}(\mathfrak{h})$ for some $\lambda \in Y(G)$, each member of which is $G$-cr.

\section{Theorem 5.26.}

(i) Let $n \in \mathbb{N}$, let $\mathbf{h} \in \mathfrak{g}^{n}$ and let $\lambda \in Y(G)$ such that $\mathbf{m}:=\lim _{a \rightarrow 0} \lambda(a) \cdot \mathbf{h}$ exists. Then the following are equivalent:

(a) $\mathbf{m}$ is $G$-conjugate to $\mathbf{h}$;

(b) $\mathbf{m}$ is $R_{u}\left(P_{\lambda}\right)$-conjugate to $\mathbf{h}$;

(c) $\operatorname{dim} G \cdot \mathbf{m}=\operatorname{dim} G \cdot \mathbf{h}$.

(ii) Let $\mathfrak{h}$ be a subalgebra of $\mathfrak{g}$ and let $\lambda \in Y(G)$. Suppose $\mathfrak{h} \subseteq \mathfrak{p}_{\lambda}$ and set $\mathfrak{m}=c_{\lambda}(\mathfrak{h})$. Then $\operatorname{dim} C_{G}(\mathfrak{m}) \geq \operatorname{dim} C_{G}(\mathfrak{h})$ and the following are equivalent:

(a) $\mathfrak{m}$ is $G$-conjugate to $\mathfrak{h}$;

(b) $\mathfrak{m}$ is $R_{u}\left(P_{\lambda}\right)$-conjugate to $\mathfrak{h}$;

(c) $\mathfrak{h}$ is contained in the Lie algebra of an $R$-Levi subgroup of $P_{\lambda}$;

(d) $\operatorname{dim} C_{G}(\mathfrak{m})=\operatorname{dim} C_{G}(\mathfrak{h})$.

(iii) Let $\mathfrak{h}, \lambda$ and $\mathfrak{m}$ be as in (ii) and let $\mathbf{h} \in \mathfrak{h}^{n}$ be a generating tuple of $\mathfrak{h}$. Then the assertions in (i) are equivalent to those in (ii). In particular, $\mathfrak{h}$ is $G$-completely reducible if and only if $G \cdot \mathbf{h}$ is closed in $\mathfrak{g}^{n}$.

Note that the final statement of Theorem [5.26(iii) is [22, Thm. 1(1)].

If $\mathfrak{h}$ is a Lie subalgebra of $\mathfrak{g}$ and $\mathfrak{h} \subseteq \mathfrak{p}_{\lambda}$ for $\lambda \in Y(G)$, then setting $\mathfrak{m}:=c_{\lambda}(\mathfrak{h})$ and $S_{n}(\mathfrak{m}):=\overline{G \cdot \mathfrak{m}^{n}}$, we get an optimal class $\Omega\left(\mathfrak{h}^{n}, S_{n}(\mathfrak{m})\right)$ of cocharacters, as in Definition 5.15

Theorem 5.27. Let $G, G^{\prime}$ and \|\| be as in Theorem 5.16. Let $\mathfrak{h}$ be any subalgebra of $\mathfrak{g}$ and let $n \in \mathbb{N}$ such that $\mathfrak{h}^{n}$ contains a generating tuple of $\mathfrak{h}$. Let $\mathfrak{m}$ be a 
subalgebra of $\mathfrak{g}$ and suppose that $\mathfrak{m}=c_{\lambda}(\mathfrak{h})$ for some $\lambda \in Y_{k}(G)$ with $\mathfrak{h} \subseteq \mathfrak{p}_{\lambda}$. Put $\Omega(\mathfrak{h}, \mathfrak{m}, k):=\Omega\left(\mathfrak{h}^{n}, S_{n}(\mathfrak{m}), k\right)$. Then the following hold:

(i) $P_{\mu}=P_{\nu}$ for all $\mu, \nu \in \Omega(\mathfrak{h}, \mathfrak{m}, k)$. Let $P(\mathfrak{h}, \mathfrak{m}, k)$ denote the unique $R$-parabolic subgroup of $G$ so defined. Then $\mathfrak{h} \subseteq \operatorname{Lie}(P(\mathfrak{h}, \mathfrak{m}, k))$ and $R_{u}(P(\mathfrak{h}, \mathfrak{m}, k))(k)$ acts simply transitively on $\Omega(\mathfrak{h}, \mathfrak{m}, k)$.

(ii) For $g \in G^{\prime}(k)$ we have $\Omega(g \cdot \mathfrak{h}, g \cdot \mathfrak{m}, k)=g \cdot \Omega(\mathfrak{h}, \mathfrak{m}, k)$ and $P(g \cdot \mathfrak{h}, g \cdot \mathfrak{m}, k)=$ $g P(\mathfrak{h}, \mathfrak{m}, k) g^{-1}$. If $g \in G(k)$ normalizes $\mathfrak{h}$ and stabilizes $S_{n}(\mathfrak{m})$, then $g \in$ $P(\mathfrak{h}, \mathfrak{m}, k)$.

(iii) If $\mu \in \Omega(\mathfrak{h}, \mathfrak{m}, k)$, then $\operatorname{dim} C_{G}\left(c_{\mu}(\mathfrak{h})\right) \geq \operatorname{dim} C_{G}(\mathfrak{m})$. If $\mathfrak{m}$ is $G$-conjugate to $\mathfrak{h}$, then $\Omega(\mathfrak{h}, \mathfrak{m}, k)=\{0\}$ and $P(\mathfrak{h}, \mathfrak{m}, k)=G$. If $\mathfrak{m}$ is not $G$-conjugate to $\mathfrak{h}$, then $\mathfrak{h}$ is not contained in the Lie algebra of any $R$-Levi subgroup of $P(\mathfrak{h}, \mathfrak{m}, k)$.

Definition 5.28. We call $\Omega(\mathfrak{h}, \mathfrak{m}, k)$ the optimal class for $\mathfrak{h}$ with respect to $\mathfrak{m}$ over $k$ and we call $P(\mathfrak{h}, \mathfrak{m}, k)$ the optimal destabilizing $R$-parabolic subgroup for $\mathfrak{h}$ with respect to $\mathfrak{m}$ over $k$. Assume the $G$-conjugacy class given by Remark 5.25] contains a subalgebra $\mathfrak{m}$ of the form $c_{\lambda}(\mathfrak{h})$ for some $\lambda \in Y_{k}(G)$. Then we set $\Omega(\mathfrak{h}, k):=$ $\Omega(\mathfrak{h}, \mathfrak{m}, k)$ and $P(\mathfrak{h}, k):=P(\mathfrak{h}, \mathfrak{m}, k)$. Under this assumption we have, by Remark 5.25 and Theorem 5.27, that $N_{G(k)}(\mathfrak{h})$ is contained in $P(\mathfrak{h}, k)$ and that for $\mu \in$ $\Omega(\mathfrak{h}, k), c_{\mu}(\mathfrak{h})$ is $G$-completely reducible. So, by Theorem 5.26(ii), if $\mathfrak{h}$ is not $G$ completely reducible, then $\mathfrak{h}$ is not contained in the Lie algebra of any R-Levi subgroup of $P(\mathfrak{h}, k)$. Note that, trivially, $P(\mathfrak{h}, k)=G$ if $\mathfrak{h}$ is $G$-completely reducible. We call $\Omega(\mathfrak{h}, k)$ the optimal class for $\mathfrak{h}$ over $k$ and we call $P(\mathfrak{h}, k)$ the optimal destabilizing $R$-parabolic subgroup for $\mathfrak{h}$ over $k$.

Note that the assumption of the previous paragraph is satisfied if $k$ is algebraically closed. In that case we usually suppress the $k$ argument and write simply $\Omega(\mathfrak{h})$ and $P(\mathfrak{h})$ instead; we refer to these as the optimal class for $\mathfrak{h}$ and the optimal destabilizing $R$-parabolic subgroup for $\mathfrak{h}$, respectively.

Example 5.29. As a further illustration of the power of our construction, we use Theorem 5.27 to give a short alternative proof of [22, Thm. 1(2)], which states that $\mathfrak{h}=$ Lie $H$ is $G$-completely reducible if $H$ is $G$-completely reducible.

Let $H$ be a subgroup of $G$. Assume that $\mathfrak{h}$ is not $G$-cr. Let $P(\mathfrak{h})$ be the optimal destabilizing R-parabolic subgroup for $\mathfrak{h}$. By Theorem $5.27\left(\right.$ ii), $N_{G}(\mathfrak{h}) \subseteq P(\mathfrak{h})$. Clearly, $H \subseteq N_{G}(\mathfrak{h})$. Moreover, if $\mu \in \Omega(\mathfrak{h})$ and $H \subseteq L_{\mu}$, then $\mathfrak{h} \subseteq \mathfrak{l}_{\mu}$. This is impossible by Theorem 5.27 (iii), so $H$ is not $G$-cr. Thus we can conclude that if $H$ is $G$-cr, then so is $\mathfrak{h}$.

5.4. A special case of the Centre Conjecture. In this final section we describe an application of optimal destabilizing parabolic subgroups to the theory of spherical buildings [36. Suppose from now on that $G$ is connected. Let $X=X(G, k)$ be the spherical Tits building of $G$ over $k$; then $X$ is a simplicial complex whose simplices correspond to the $k$-defined parabolic subgroups of $G$. The conjugation action of $G(k)$ on itself naturally induces an action of $G(k)$ on $X$. We identify $X$ with its geometric realization. A subcomplex $Y$ of $X$ is convex if whenever two points of $Y$ are not opposite in $X$, then $Y$ contains the unique geodesic joining these points, and $Y$ is contractible if it has the homotopy type of a point. The following is a version due to Serre of the so-called Centre Conjecture of J. Tits 34 , Sec. 2.4]. This has been proved by B. Mühlherr and J. Tits for spherical buildings of classical type [23]. 
Conjecture 5.30. Let $Y$ be a convex and contractible subcomplex of $X$. Then there is a point $y \in Y$ such that $y$ is fixed by any automorphism of $X$ that stabilizes $Y$.

A point $y \in Y$ whose existence is asserted in Conjecture 5.30 is frequently referred to as a "natural centre" or just "centre" of $Y$. Our idea is to take as a centre of $Y$ the barycentre of the simplex corresponding to the optimal destabilizing parabolic subgroup in an appropriate sense. This approach is not new; indeed, it was part of the motivation for Kempf's paper [15] on optimality (cf. [24, p. 64]). We show how to make this work to prove the Centre Conjecture in the case that $Y$ is the fixed point subcomplex $X^{H}$ for some subgroup $H$ of $G$, where $X^{H}$ consists of all the simplices in $X$ corresponding to parabolic subgroups containing $H$. Note that $X^{H}$ is always a convex subcomplex of $X[32, \S 2.3 .1]$.

Theorem 5.31. Suppose $G$ is semisimple and adjoint and $k$ is a perfect field. Let $H$ be a subgroup of $G$ and suppose that $Y:=X^{H}$ is contractible. Then there is a point $y \in Y$ which is fixed by any element of (Aut $G)(k)$ that stabilizes $Y$.

Proof. Since we are assuming that $G$ is semisimple and defined over $k$, Aut $G$ is an algebraic group also defined over $k$ [36, 5.7.2]. Since $G$ is adjoint, we can also view $G$ as a subgroup of Aut $G$. Let $K$ be the intersection of all the $k$-defined parabolic subgroups of $G$ that contain $H$. Then $K$ is $k$-defined, because $k$ is perfect, and $X^{K}=Y$; cf. the proof of [3, Thm. 3.1]. Since $Y$ is contractible, $K$ is not $G$-cr over $k$, by a result of Serre [34, Sec. 3], and hence $K$ is not $G$-cr, by [1, Thm. 5.8].

Now let $M$ be a representative of the unique $G$-conjugacy class of $G$-cr subgroups attached to $K$ given by Proposition 5.14 Let $P=P(K)$ be the optimal destabilizing parabolic subgroup for $K$ (over $\bar{k}$ ), Definition 5.17. Then $P$ is a parabolic subgroup of $G$ containing $K$, by Theorem 5.16(i), and $P$ is defined over $k$, by Theorem 5.18 (ii), so $P$ corresponds to a simplex of $Y$. Moreover, any element of (Aut $G)(k)$ that stabilizes $Y$ also normalizes $K$, and hence stabilizes the $G$-conjugacy class of $M$, by Proposition 5.14(ii). So any such automorphism normalizes $P$, by Theorem 5.16(ii), with $G^{\prime}=\operatorname{Aut} G$. We can therefore take $y$ to be the barycentre of the simplex corresponding to $P$.

Remark 5.32. The assumptions that $G$ is semisimple and adjoint in Theorem 5.31 allow us to apply our optimality results, because they ensure that Aut $G$ is an algebraic group and $G$ is a subgroup of Aut $G$. In the context of buildings, however, these assumptions are no loss: given any connected reductive $G$, let Ad denote the adjoint representation of $G$. Then the building of $G$ is isomorphic to the building of the adjoint group $\operatorname{Ad}(G)$, and a subgroup $H$ of $G$ is $G$-cr if and only if the image of $H$ in $\operatorname{Ad}(G)$ is $\operatorname{Ad}(G)$-cr [1, Lem. 2.12]. Moreover, all automorphisms of $X(G)$ that come from Aut $G$ survive this transition from $G$ to $\operatorname{Ad}(G)$.

To establish that the Centre Conjecture holds for subcomplexes of the form $Y=$ $X^{H}$, we need to find a centre $y \in Y$ which is fixed by all building automorphisms of $X$ that stabilize $X^{H}$, not just the building automorphisms that arise from algebraic automorphisms of $G$. For most $G$, however, Aut $X$ is generated by Aut $G$ together with field automorphisms; see [36, Cor. 5.9] for more details. We finish by showing how to deal with field automorphisms in some cases.

Recall that $\Gamma$ denotes the group $\operatorname{Gal}\left(k_{s} / k\right)$. Following [36, 5.7.1], any $\gamma \in \Gamma$ induces an automorphism of the building $X=X(G, \bar{k})$, which we also denote by 
$\gamma$. Recall that $\Gamma$ also acts on the set of cocharacters $Y(G)$ and we can ensure that the norm is invariant under this action (i.e., the norm is $k$-defined in the sense of Section 4).

Theorem 5.33. Suppose $G$ is connected. Let $X=X(G, \bar{k})$ be the building of $G$ over the algebraic closure of $k$. Let $H$ be a subgroup of $G$ and suppose that $Y:=X^{H}$ is contractible. Let $\Gamma_{Y}$ denote the subgroup of $\Gamma$ that stabilizes $Y$. Then there is a point $y \in Y$ which is fixed by any element of $\Gamma_{Y}$.

Proof. As in the proof of Theorem 5.31, let $K$ be the intersection of the parabolic subgroups corresponding to simplices in $Y$. Then $Y=X^{K}$, and since $Y$ is stabilized by all $\gamma \in \Gamma_{Y}$, we have $\gamma \cdot K=K$ for all $\gamma \in \Gamma_{Y}$. Let $\lambda$ and $M=c_{\lambda}(K)$ be as in Proposition 5.14. Choose $n \in \mathbb{N}$ such that $K$ admits a generic $n$-tuple $\mathbf{k} \in K^{n}$. Then $S_{n}(M)$ is $\Gamma_{Y}$-stable by Proposition 5.14 (iii). Because the norm is $\Gamma$-invariant, for any $\lambda \in \Lambda\left(K^{n}\right)$ and any $\gamma \in \Gamma_{Y}$, we have

$$
\frac{\alpha_{S_{n}(M), K^{n}}(\gamma \cdot \lambda)}{\|\gamma \cdot \lambda\|}=\frac{\alpha_{S_{n}(M), K^{n}}(\lambda)}{\|\lambda\|} .
$$

It follows that the optimal parabolic subgroup $P(K)$ for $K$ is stabilized by $\Gamma_{Y}$. We can therefore take $y$ to be the barycentre of the simplex corresponding to $P(K)$.

Remarks 5.34. (i). Combining Theorem 5.31 and Theorem 5.33 goes a long way towards proving the full version of Tits' Centre Conjecture for subcomplexes of the form $X^{H}$ in many cases. For example, if $G$ is a split simple group of adjoint type defined over a finite field $k$, then, with a few exceptions, the automorphism group of $X(G, \bar{k})$ is a split extension of Aut $G$ by the automorphism group of the field $\bar{k}$ (see [36. Cor. 5.10]), and the results above show how to deal with many of these automorphisms.

(ii). Theorems 5.31 and 5.33 improve on [3, Thm. 3.1].

\section{ACKNOWLEDGEMENTS}

The authors acknowledge the financial support of EPSRC Grant EP/C542150/1, Marsden Grant UOC0501 and the DFG-priority programme SPP1388 "Representation Theory". Part of the research for this paper was carried out while the authors were staying at the Mathematical Research Institute Oberwolfach supported by the "Research in Pairs" programme. Also, part of this paper was written during a stay of the first three authors at the Isaac Newton Institute for Mathematical Sciences, Cambridge during the "Algebraic Lie Theory" Programme in 2009. Finally, we are grateful to the referee for carefully reading the manuscript and for some suggestions.

\section{REFERENCES}

[1] M. Bate, B. Martin, G. Röhrle, A geometric approach to complete reducibility, Invent. Math. 161, no. 1 (2005), 177-218. MR2178661 (2007k:20101)

[2] - Complete reducibility and commuting subgroups, J. Reine Angew. Math. 621, (2008), 213-235. MR2431255 (2009h:20051)

[3] - On Tits' Centre Conjecture for fixed point subcomplexes, C. R. Acad. Sci. Paris Ser. I Math. 347, (2009) 353-356. MR2537229 (2010i:20049)

[4] M. Bate, B. Martin, G. Röhrle, R. Tange, Complete reducibility and separability, Trans. Amer. Math. Soc., 362, no. 8, (2010), 4283-4311. MR2608407(2011i:20068)

[5] Complete reducibility and conjugacy classes of tuples in algebraic groups and Lie algebras, Math. Z., 269, no. 3-4 (2011), 809-832. MR2860266 
[6] A. Borel, Properties and linear representations of Chevalley groups, Seminar on Algebraic Groups and Related Finite Groups, pp. 1-55, Lecture Notes in Mathematics, Vol. 131, Springer, Berlin, 1970. MR0258838 (41:3484)

[7] Linear algebraic groups, Graduate Texts in Mathematics, 126, Springer-Verlag, 1991. MR 1102012 (92d:20001)

[8] A. Borel, J. Tits, Éléments unipotents et sous-groupes paraboliques des groupes réductifs, I, Invent. Math. 12 (1971), 95-104. MR0294349(45:3419)

[9] _ Compléments à l'article: “Groupes réductifs”, Inst. Hautes Études Sci. Publ. Math. No. 41 (1972), 253-276. MR0315007 (47:3556)

[10] C.W. Curtis, I. Reiner, Representation theory of finite groups and associative algebras. Pure and Applied Mathematics, Vol. XI, Interscience, John Wiley \& Sons, New York-London, 1962. MR0144979(26:2519)

[11] T.L. Gómez, A. Langer, A.H.W. Schmitt, I. Sols, Moduli spaces for principal bundles in arbitrary characteristic. Adv. Math. 219, no. 4 (2008), 1177-1245. MR2450609 (2009j:14014)

[12] W.H. Hesselink, Uniform instability in reductive groups, J. Reine Angew. Math. 303/304 (1978), 74-96. MR.514673 (81a:14022)

[13] _ Desingularizations of varieties of nullforms, Invent. Math. 55 (1979), no. 2, 141-163. MR.553706 (81b:14025)

[14] J.C. Jantzen, Nilpotent orbits in representation theory, in: Lie theory: Lie algebras and representations, Progress in Math. 228, Birkhaüser Boston (2004). MR2042689 (2005c:14055)

[15] G.R. Kempf, Instability in invariant theory, Ann. of Math. (2) 108 (1978), 299-316. MR.506989 (80c:20057)

[16] J. Levy, Rationality and orbit closures, Canad. Math. Bull. 46, (2003), 204-215. MR.1981675 (2004c:14090)

[17] M.W. Liebeck, G.M. Seitz, Reductive subgroups of exceptional algebraic groups. Mem. Amer. Math. Soc. no. 580 (1996). MR1329942 (96i:20059)

[18] _ Variations on a theme of Steinberg, Special issue celebrating the 80th birthday of Robert Steinberg. J. Algebra 260 (2003), no. 1, 261-297. MR1973585 (2004g:20064)

[19] M.W. Liebeck, D.M. Testerman, Irreducible subgroups of algebraic groups, Q. J. Math. 55 (2004), 47-55. MR2043006 (2005b:20087)

[20] B. Martin, Reductive subgroups of reductive groups in nonzero characteristic, J. Algebra 262 (2003), no. 2, 265-286. MR.1971039 (2004g:20066)

[21] _ A normal subgroup of a strongly reductive subgroup is strongly reductive, J. Algebra 265 (2003), no. 2, 669-674. MR1987023 (2004e:20080)

[22] G. McNinch, Completely reducible Lie subalgebras, Transform. Groups 12 (2007), no. 1, 127135. MR2308032(2008a:17022)

[23] B. Mühlherr, J. Tits, The Centre Conjecture for non-exceptional buildings, J. Algebra 300 (2006), no. 2, 687-706. MR.2228217 (2007e:51018)

[24] D. Mumford, J. Fogarty, F. Kirwan, Geometric invariant theory. Third edition. Ergebnisse der Mathematik und ihrer Grenzgebiete, 34. Springer-Verlag, Berlin, 1994. MR1304906 (95m:14012)

[25] P. E. Newstead, Introduction to moduli problems and orbit spaces, published for the Tata Institute of Fundamental Research, Bombay, by Springer-Verlag, 1978. MR.546290 (81k:14002)

[26] A. Premet, Nilpotent orbits in good characteristic and the Kempf-Rousseau theory, J. Algebra 260 (2003), no. 1, 338-366. MR1976699 (2004i:17014)

[27] R.W. Richardson, Conjugacy classes of $n$-tuples in Lie algebras and algebraic groups, Duke Math. J. 57 (1988), no. 1, 1-35. MR952224 (89h:20061)

[28] G. Rousseau, Immeubles sphériques et théorie des invariants, C.R.Acad. Sci. Paris Sér. A-B 286 (1978), 247-250. MR0506257 (58:22063)

[29] A.H.W. Schmitt, A closer look at semistability for singular principal bundles. Int. Math. Res. Not. 62 (2004), 3327-3366. MR2097106 (2005h:14105)

[30] G.M. Seitz, Abstract homomorphisms of algebraic groups, J. London Math. Soc. (2) 56 (1997), no. 1, 104-124. MR1462829 (99b:20077)

[31] J.-P. Serre, Semisimplicity and tensor products of group representations: converse theorems. With an appendix by Walter Feit, J. Algebra 194 (1997), no. 2, 496-520. MR1467165 (98i:20008)

[32] L La notion de complète réductibilité dans les immeubles sphériques et les groupes réductifs, Séminaire au Collège de France, résumé dans [37, pp. 93-98.] (1997). 
[33] - The notion of complete reducibility in group theory, Moursund Lectures, Part II, University of Oregon, 1998, arXiv:math/0305257v1 [math.GR].

[34] _ Complète réductibilité, Séminaire Bourbaki, 56ème année, 2003-2004, no 932. MR2167207 (2006d:20084)

[35] T.A. Springer, Linear algebraic groups, Second edition. Progress in Mathematics, 9. Birkhäuser Boston, Inc., Boston, MA, 1998. MR:1642713(99h:20075)

[36] J. Tits, Buildings of spherical type and finite BN-pairs, Lecture Notes in Math. 386, Springer-Verlag (1974). MR0470099 (57:9866)

[37] _ Théorie des groupes, Résumé des Cours et Travaux, Annuaire du Collège de France, 97 ${ }^{\mathrm{e}}$ année, (1996-1997), 89-102. MR1320558

Department of Mathematics, University of York, York YO10 5DD, United Kingdom E-mail address: michael.bate@york.ac.uk

Mathematics and Statistics Department, University of Canterbury, Private Bag 4800, Christchurch 1, New Zealand

E-mail address: B.Martin@math.canterbury.ac.nz

Current address: Department of Mathematics, University of Auckland, Private Bag 92019, Auckland 8140, New Zealand

E-mail address: Ben.Martin@auckland.ac.nz

FAkUltät Für Mathematik, Ruhr-Universität Bochum, D-44780 Bochum, Germany

E-mail address: gerhard.roehrle@rub.de

School of Mathematics, Trinity College Dublin, College Green, Dublin 2, Ireland E-mail address: tanger@tcd.ie

Current address: College of Engineering, Mathematics and Physical Sciences, University of Exeter, Exeter, EX4 4QF, United Kingdom

E-mail address: R.Tange@exeter.ac.uk 